\title{
Low-Reynolds-number gravity-driven migration and deformation of bubbles near a free surface
}

\author{
Franck Pigeonneau ${ }^{1}$ and Antoine Sellier ${ }^{2}$ \\ ${ }^{1}$ Surface du Verre et Interfaces - UMR 125 CNRS/Saint-Gobain, 39 Quai Lucien Lefranc - BP 135, \\ 93303 Aubervilliers Cedex, France \\ ${ }^{2}$ Laboratoire d'Hydrodynamique (LadHyX), Ecole Polytechnique, 91128 Palaiseau Cedex, France
}

(Received 18 January 2011; accepted 18 July 2011; published online 6 September 2011)

\begin{abstract}
We investigate numerically the axisymmetric migration of bubbles toward a free surface, using a boundary-integral technique. Our careful numerical implementation allows to study the bubble(s) deformation and film drainage; it is benchmarked against several tests. The rise of one bubble toward a free surface is studied and the computed bubble shape compared with the results of Princen [J. Colloid Interface Sci. 18, 178 (1963)]. The liquid film between the bubble and the free surface is found to drain exponentially in time in full agreement with the experimental work of Debrégeas et al. [Science 279, 1704 (1998)]. Our numerical results also cast some light on the role played by the deformation of the fluid interfaces and it turns out that for weakly deformed interfaces (high surface tension or a tiny bubble) the film drainage is faster than for a large fluid deformation. By introducing one or two additional bubble(s) below the first one, we examine to which extent the previous trends are affected by bubble-bubble interactions. For instance, for a 2-bubble chain, decreasing the bubblebubble separation increases the deformation of the last bubble in the chain. Finally, the exponential drainage of the film between the free surface and the closest bubble is preserved, yet the drainage is enhanced. (C) 2011 American Institute of Physics. [doi:10.1063/1.3629815]
\end{abstract}

\section{INTRODUCTION}

Phase separation is involved in many chemical processes, such as flotation, liquid-liquid or gas-liquid extractions. The final stage of such processes, in which two phases have to be separated, is generally limited by the collapse of inclusions at the free surface. For instance, the coalescence of bubbles in highly viscous fluids is observed in various fields, such as geophysics ${ }^{1}$ or the glass industry. For the latter application, the energetic efficiency of glass furnaces is deeply related to the occurrence of a foam layer on top of the molten glass bath. ${ }^{2}$ Since the transition between frost and foam depends strongly upon the lifetime of a bubble in the vicinity of the free surface, more insights into the idealized problem of a drop or a bubble moving toward a free surface are needed in order to handle such basic and industrial issues.

Earlier basic studies in this direction include the work of Princen, ${ }^{3}$ who approximated the drop shape at a liquid-liquid free interface by balancing the pressures driven by gravity with surface tension effects. Hartland ${ }^{4}$ confirmed experimentally the theoretical predictions of Princen, ${ }^{3}$ using a drop made of glycerol or golden syrup immersed in liquid paraffin (light fluid). Hartland ${ }^{5}$ also determined the profile of the film thickness and observed a minimum film thickness near the edge of the film. $\mathrm{He}^{6}$ later developed a theoretical model based on lubrication theory, in which faster drainage occurred for mobile interfaces. Jones and Wilson ${ }^{7}$ developed a lubrication theory to determine the behavior of the liquid film where the settling of a solid particle and a drop were both studied. They demonstrated that the film thickness behaves as an algebraic function of time.

The special case of bubble drainage in a high viscous fluid was experimentally examined by Debrégeas et al. ${ }^{8}$ using silicon oil. From an interferometry method, an exponential decrease of the film thickness with time was clearly established. This study was limited to large bubbles compared to the capillary scale. Howell ${ }^{9}$ later studied the drainage of a bubble close to a free surface, using a lubrication model and restricting attention to a bidimensional bubble. Nevertheless, the case of axisymmetric bubble was reported in an appendix where Howell showed that the film thickness evolves as an algebraic function of time when the deformation is small, and the gravity is neglected.

One drawback of the lubrication theory is the poor knowledge of the initial conditions. Generally, the latter depend on the dynamics prior to the lubrication regime. With this idea in mind, Chi and Leal ${ }^{10}$ developed a numerical method based on an integral formulation of Stokes equations in axisymmetric configuration. This work has been done for a viscous drop with a dynamic viscosity that is a multiple $\hat{\mu}$ of the viscosity of the continuous liquid, and computations were achieved mainly for $\hat{\mu}$ in the domain $[0.1,10]$. Chi and Leal ${ }^{10}$ pointed out a fast drainage for small viscosity ratio $\hat{\mu}$, a neutral drainage for $\hat{\mu}=\mathcal{O}(1)$ and a low drainage for large $\hat{\mu}$. A boundary-integral equation approach has been also used by Manga ${ }^{1}$ to study how a drop crosses a liquidliquid interface.

Two-phase flows can be numerically studied by various techniques. The oldest one is the "Volume of Fluid" method pioneered by Hirt and Nichols, ${ }^{11}$ where the two phases are seen as a single fluid with a concentration varying between 0 in a phase and 1 in another one. Interfaces are then tracked setting this concentration equal to $1 / 2$. The "level set" approach is based on the description of a distance function from an interface where the interface is mathematically defined for the level set equal to zero. This method is for 
example used by Sussman et al. ${ }^{12}$ Another method is the "front-tracking" technique in which the interfaces between the two phases are tracked using a discrete representation of the interfaces (see, for instance, Unverdi et al. ${ }^{13}$ ). The main advantage of these numerical methods is the ability to deal with arbitrary Reynolds numbers. Unfortunately, such techniques require to discretize the entire domain and also to consider interfaces with thickness of order of a few cells. Hence, interfaces that are too close can be merged depending on the spatial resolution. This prohibits the accurate simulation of film drainage at small scales.

The boundary-integral method is a powerful alternative method, in which the fluid interfaces are carefully described. The method is based on integral formulations exploiting ${ }^{14}$ the existence of a fundamental solution (Green's functions) and of a reciprocity relationship. These requirements are fulfilled for Stokes equations, for which the reciprocity relationship is known since the pioneering work of Lorentz, ${ }^{15}$ whereas the Green's functions has been obtained 80 years ago by Odqvist. ${ }^{16}$ Theoretical issues for Stokes flows together with the associated boundary-integral equations have also been mathematically addressed by Ladyzhenskaya. ${ }^{17}$ However, the first numerical resolutions of boundary integral equations for creeping flows were only first performed by Youngren and Acrivos ${ }^{18}$ for a solid body and by Rallison and Acrivos ${ }^{19}$ for a viscous drop. As this method is now widely employed, we refer the reader to the books of Pozrikidis $^{20}$ and Kim and Karrila ${ }^{21}$ for further details.

This work examines the motion and deformation of bubbles moving toward a free surface in a highly viscous fluid when the inertial forces are negligible. Since arbitrary and fully three-dimensional geometries would result in very involved numerical implementation and computations, we restrict the study to axisymmetric geometries. This assumption therefore prevents us from tracking in time non-axisymmetric shapes disturbances that might be produced by non-axisymmetric instabilities. A boundary-integral approach of Stokes equations and a relevant well-posed boundary-integral equation specifically developed for axisymmetric geometries involving several bubbles and a free surface are obtained in Sec. III. Since we aim at investigating the film drainage between bubbles or bubble and a free surface, special efforts are made to solve accurately the boundary-integral equations, as detailed in Sec. IV. The implemented procedure then permits us to study the motion and drainage of one, two, and three bubbles in axisymmetric configuration in Sec. V.

\section{GOVERNING EQUATIONS AND RELEVANT PROCEDURE}

We consider a Newtonian liquid with uniform viscosity $\mu$ and density $\rho$, subject to the uniform vertical gravity field $\boldsymbol{g}=-g \boldsymbol{e}_{3}$ with magnitude $g>0$. The ambient fluid above the $z=0$ plane free surface is a gas with a uniform pressure $p_{a}$. Injecting $N \geq 1$ bubble(s) in the liquid results, by buoyancy effects, in a motion of each bubble toward the free surface, whereas the shape of the bubble(s) and the free surface both change with time. As outlined in the introduction, determining those time-dependent shapes exhibiting significant deformations

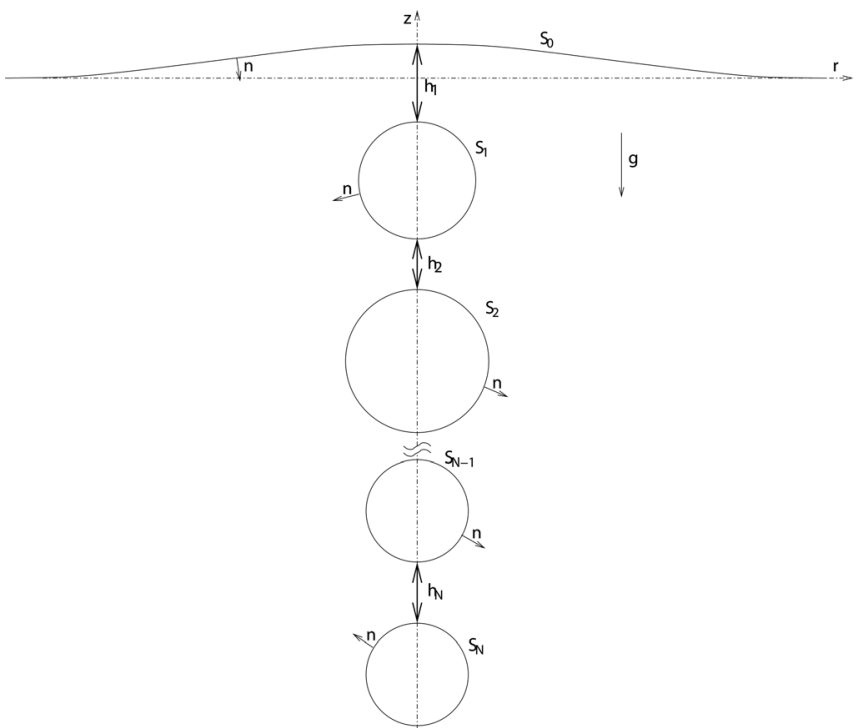

FIG. 1. Retained axisymmetric configurations for $N \geq 1$ bubble(s).

is a challenging issue of fundamental interest. The present work addresses such a task for axisymmetric configurations. As depicted in Fig. 1, one therefore assumes at each time a free surface and a single bubble or a $N$-bubble chain (for $N \geq 2$ ) that share an axis of revolution parallel to the gravity field $g$. The different surfaces are numbered from the top to the bottom with bubble $\mathcal{B}_{n}$ having a smooth surface $S_{n}$, whereas $S_{0}$ denotes the disturbed free surface. Moreover, those boundaries are characterized by the same constant surface tension $\gamma$, and a unit normal vector $\boldsymbol{n}$ directed into the liquid.

Each bubble $\mathcal{B}_{n}$, made of a gas with uniform pressure $p_{n}$ (for instance) and negligible density and viscosity, is spherical with radius $a_{n}$ at initial time. In the time-dependent liquid domain $\mathcal{D}(t)$, the fluid has pressure $p+\rho \boldsymbol{g} \cdot \boldsymbol{x}$ and velocity $\boldsymbol{u}$ with typical magnitude $U=\rho g a^{2} /(3 \mu)$, where $a=\max \left(a_{n}\right)$. Moreover, the resulting Reynolds number Re obeys

$$
\operatorname{Re}=\rho U a / \mu \ll 1,
$$

so that all inertial effects are neglected. Assuming quasisteady changes for the bubble(s) and free surface shapes, the flow $(\boldsymbol{u}, p)$ therefore fulfills the steady Stokes equations

$$
\begin{gathered}
\mu \nabla^{2} \boldsymbol{u}=\operatorname{grad} p, \\
\nabla \cdot \boldsymbol{u}=0,
\end{gathered}
$$

in the liquid domain $\mathcal{D}(t)$, to be supplemented with the farfield behavior

$$
(\boldsymbol{u}, p) \rightarrow(\mathbf{0}, 0), \quad \text { as }\|\boldsymbol{x}\| \rightarrow \infty,
$$

and additional boundary conditions on each surface $S_{n}$ for $n=0, \ldots, N$. Setting $p_{0}=p_{a}$ and denoting by $\boldsymbol{\sigma}$, the stress tensor of the flow $(\boldsymbol{u}, p)$, these relevant conditions consist of both the relation (because the surface tension $\gamma$ is uniform)

$$
\boldsymbol{\sigma} \cdot \boldsymbol{n}=\left(\rho \boldsymbol{g} \cdot \boldsymbol{x}-p_{n}+\gamma \nabla_{S} \cdot \boldsymbol{n}\right) \boldsymbol{n},
$$

on $S_{n}$ for $n=0, \ldots, N$, and the usual zero-mass flux conditions (impermeable surfaces) 


$$
V \cdot n=u \cdot n,
$$

on $S_{n}$ for $n=0, \ldots, N$, where $\boldsymbol{V}$ is the material velocity on each surface $S_{n}$. In Eq. (5), the quantity $\nabla_{S} \cdot \boldsymbol{n}$ is the surface divergence of the unit normal $\boldsymbol{n}$, which is related to the local average curvature $H$ as $\nabla_{S} \cdot \boldsymbol{n}=2 H$ (see Aris $^{22}$ ). Furthermore, we assume in practice that both the temperature and the pressure are uniform and time independent in each bubble, which its volume is therefore constant as time evolves. Under the boundary conditions (6), one then supplements Eqs. (2)-(5) with the additional relations

$$
\int_{S_{n}} \boldsymbol{u} \cdot \boldsymbol{n} d S=0, \quad \text { for } n=1, \ldots, N .
$$

Observe that Eq. (7) also holds on the free surface $S_{0}$ because $\boldsymbol{u}$ is divergence free, see Eq. (3), and Eq. (7) is valid for each bubble.

At that stage, it is worth highlighting the following steps when tracking in time the shapes of the bubble(s) and free surface:

At a given time $t$, one first obtains the liquid flow $(\boldsymbol{u}, p)$ in the given fluid domain $\mathcal{D}(t)$ with boundaries $S_{n}$ by solving the problem (2)-(5) in conjunction with Eq. (7). As explained in Sec. III B, one then actually gets a unique solution ( $\boldsymbol{u}, p)$ for Eqs. (2)-(5) and (7).

(ii) Once $(\boldsymbol{u}, p)$ has been obtained, one gets at the same time $t$ the normal component $\boldsymbol{V} \cdot \boldsymbol{n}$ by exploiting Eq. (6). The knowledge of this normal velocity $\boldsymbol{V} \cdot \boldsymbol{n}$ then permits one to move each bubble and the free surface for a given time step $d t$ and then to determine the updated liquid domain $\mathcal{D}(t+d t)$.

The entire procedure (i)-(ii) is embedded in a RungeKutta algorithm to determine the time-dependent shapes of the bubble(s) and free surface. Such a scheme, quite simple by essence, however, deserves a few key remarks:

(i) It requires to efficiently solve at each time the wellposed problem (2)-(5) and (7). At a first glance, this might be numerically achieved by computing the flow $(\boldsymbol{u}, p)$ in the entire liquid domain using a standard finite element technique for instance. Unfortunately, this would first require to adequately truncate the unbounded liquid domain and it would also become very cpu-time consuming if a good accuracy is required. Another boundary approach, free from these important drawbacks, is therefore introduced and implemented in Secs. III and IV.

(ii) As previously noticed, the key boundary condition (5) involves the local curvature $H=\nabla_{S} \cdot \boldsymbol{n} / 2$ on each boundary. Clearly, the accuracy level at which this quantity is numerically approximated directly dictates the accuracy of the whole method and one therefore needs to adequately discretize, as detailed in Sec. IV, each surface $S_{n}$.

(iii) In order to accurately track in time the drainage occurring for small bubble-bubble or/and bubble free-surface gap(s), one must resort to a careful numerical treatment of the boundary integrals and use as many nodal points as necessary.

\section{BOUNDARY FORMULATION}

We present in this section the advocated boundary approach to accurately solve the problem (2)-(5) and (7) for a given liquid domain $\mathcal{D}(t)$ and prescribed gravity field $\boldsymbol{g}$, uniform surface tension $\gamma$, uniform ambient pressure $p_{a}$, and uniform pressures $p_{n}$ for $n=1, \ldots, N$.

\section{A. Relevant integral representation and associated boundary-integral equation}

Since the flow $(\boldsymbol{u}, p)$ obeys in the liquid domain the Stokes equations (2) and the far-field behavior (4), its velocity field $\boldsymbol{u}$ receives in the entire liquid domain the following widely employed integral representation: ${ }^{20}$

$$
\begin{aligned}
\boldsymbol{u}\left(\boldsymbol{x}_{0}\right)= & -\frac{1}{8 \pi \mu} \int_{S} \boldsymbol{f}(\boldsymbol{x}) \cdot \boldsymbol{G}\left(\boldsymbol{x}, \boldsymbol{x}_{0}\right) d S(\boldsymbol{x}) \\
& +\frac{1}{8 \pi} \int_{S} \boldsymbol{u}(\boldsymbol{x}) \cdot \boldsymbol{T}\left(\boldsymbol{x}, \boldsymbol{x}_{0}\right) \cdot \boldsymbol{n}(\boldsymbol{x}) d S(\boldsymbol{x}),
\end{aligned}
$$

with the entire surface $S=\cup_{n=0}^{N} S_{n}$ and $\boldsymbol{f}$ the surface traction defined as

$$
f(x)=\sigma \cdot n(x),
$$

and second- and third-rank tensors $\boldsymbol{G}$ and $\boldsymbol{T}$ the usual freespace Oseen-Burgers tensor and associated stress tensor admitting the Cartesian components $G_{i j}$ and $T_{i j k}$ given by

$$
\begin{aligned}
G_{i j}\left(\boldsymbol{x}, \boldsymbol{x}_{0}\right) & =\frac{\delta_{i j}}{\left\|\boldsymbol{x}-\boldsymbol{x}_{0}\right\|}+\frac{\left(x_{i}-x_{0, i}\right)\left(x_{j}-x_{0, j}\right)}{\left\|\boldsymbol{x}-\boldsymbol{x}_{0}\right\|^{3}}, \\
T_{i j k}\left(\boldsymbol{x}, \boldsymbol{x}_{0}\right) & =-6 \frac{\left(x_{i}-x_{0, i}\right)\left(x_{j}-x_{0, j}\right)\left(x_{k}-x_{0, k}\right)}{\left\|\boldsymbol{x}-\boldsymbol{x}_{0}\right\|^{5}},
\end{aligned}
$$

where $\delta_{i j}$ is the Kronecker symbol.

Because Eq. (8) holds for $\boldsymbol{x}_{0}$ located in the liquid domain, it permits one to compute the velocity field $\boldsymbol{u}$ in the liquid by solely appealing to two surface quantities: the velocity $\boldsymbol{u}$ and the traction $\boldsymbol{f}$ on surface $S$. For the flow $(\boldsymbol{u}, p)$ governed by Eqs. (2)-(5), the traction $\boldsymbol{f}$ is prescribed by the boundary condition (5), and one therefore solely needs to determine the velocity $\boldsymbol{u}$ on the entire liquid boundary $S$. This is achieved by letting $\boldsymbol{x}_{0}$ tend to $S$ in Eq. (8). Curtailing the details which are available for instance in Pozrikidis, ${ }^{20}$ one then arrives at the following key Fredholm boundaryintegral equation of the second kind for the unknown velocity $\boldsymbol{u}$ on the liquid boundary $S$ :

$$
\begin{gathered}
4 \pi \boldsymbol{u}\left(\boldsymbol{x}_{0}\right)-f_{S} \boldsymbol{u}(\boldsymbol{x}) \cdot \boldsymbol{T}\left(\boldsymbol{x}, \boldsymbol{x}_{0}\right) \cdot \boldsymbol{n}(\boldsymbol{x}) d S(\boldsymbol{x}) \\
=-\frac{1}{\mu} \int_{S} \boldsymbol{f}(\boldsymbol{x}) \cdot \boldsymbol{G}\left(\boldsymbol{x}, \boldsymbol{x}_{0}\right) d S(\boldsymbol{x}),
\end{gathered}
$$

for $\boldsymbol{x}_{0}$ on $S$. In Eq. (12), the symbol means a weakly singular integration in the principal value sense of Cauchy (see Hadamard $^{23}$ and Kupradze ${ }^{24}$ ). It turns out that the resulting integral is actually a regular one, because of the scalar product with the unit normal $\boldsymbol{n}$. Noting that for $\boldsymbol{x}_{0}$ located on $S$ $\left(\right.$ see Pozrikidis ${ }^{20}$ ), 


$$
\int_{S_{n}} \boldsymbol{n}(\boldsymbol{x}) \cdot \boldsymbol{G}\left(\boldsymbol{x}, \boldsymbol{x}_{0}\right) d S(\boldsymbol{x})=\mathbf{0},
$$

for $n=1, \ldots, N$ and injecting the boundary condition (5) in (12) with each pressure $p_{n}$ being uniform then finally yields the boundary-integral equation

$$
\boldsymbol{u}\left(\boldsymbol{x}_{0}\right)-\frac{1}{4 \pi \mu} f_{S} \boldsymbol{u}(\boldsymbol{x}) \cdot \boldsymbol{T}\left(\boldsymbol{x}, \boldsymbol{x}_{0}\right) \cdot \boldsymbol{n}(\boldsymbol{x}) d S(\boldsymbol{x})=\boldsymbol{S}\left(\boldsymbol{x}_{0}\right),
$$

for $\boldsymbol{x}_{0}$ on $S$ and $\boldsymbol{S}\left(\boldsymbol{x}_{0}\right)$ given by

$$
\boldsymbol{S}\left(\boldsymbol{x}_{0}\right)=\frac{1}{4 \pi} \int_{S}\left[\left(\rho \boldsymbol{g} \cdot \boldsymbol{x}+\gamma \nabla_{S} \cdot \boldsymbol{n}\right) \boldsymbol{n}\right](\boldsymbol{x}) \cdot \boldsymbol{G}\left(\boldsymbol{x}, \boldsymbol{x}_{0}\right) d S(\boldsymbol{x}) .
$$

Of course (5)-(15) is recovered by setting to zero the drop viscosity ratio in Rallison and Acrivos. ${ }^{19}$ In summary, for the present work, one has to invert at each time the above boundary-integral equation (14) in conjunction with the relations (7) when the traction $\boldsymbol{f}=\boldsymbol{\sigma} \cdot \boldsymbol{n}$ is prescribed by Eq. (5). Once this is done numerically, the liquid domain is subsequently updated by employing (6) and, if necessary, the velocity field $\boldsymbol{u}$ is computed in the entire liquid domain by appealing to the integral representation (8). Since this approach only involves the surface quantities $\boldsymbol{u}$ and $\boldsymbol{\sigma} \cdot \boldsymbol{n}$, it is termed a boundary approach. It clearly solely requires to mesh the entire surface $S$ and permits one to accurately obtain the velocity $\boldsymbol{u}$ on $S$ without calculating the liquid flow $(\boldsymbol{u}, p)$ in the unbounded liquid domain.

\section{B. Basic issues for the proposed boundary approach}

Any solution $(\boldsymbol{u}, p)$ to Eqs. (2)-(5) obeys Eq. (14), but we also require $\boldsymbol{u}$ to satisfy Eq. (7). Actually, Eq. (14) does not admit a unique solution, i.e., is ill-posed. To clarify this issue, let us introduce for a given bubble $\mathcal{B}_{n}$ the eigenvalues $\lambda$ and associated eigenfunctions $\boldsymbol{v}$ defined on the bubble boundary $S_{n}$ such that

$$
\frac{1}{4 \pi} \int_{S_{n}} \boldsymbol{v}(\boldsymbol{x}) \cdot \boldsymbol{T}\left(\boldsymbol{x}, \boldsymbol{x}_{0}\right) \cdot \boldsymbol{n}(\boldsymbol{x}) d S(\boldsymbol{x})=\lambda \boldsymbol{v}\left(\boldsymbol{x}_{0}\right)
$$

for $\boldsymbol{x}_{0}$ on $S_{n}$. Whatever the bubble $\mathcal{B}_{n}$, the set of eigenvalues (the so-called spectrum) is the segment $[-1,1]$ with $\lambda=1$ having multiplicity one (see, for instance, Pozrikidis ${ }^{20}$ ). The associated normalized eigenfunctions defined on $S_{n}$ are denoted by $\boldsymbol{v}_{n}$ and such that $\int_{S_{n}} \boldsymbol{v}_{n} \cdot \boldsymbol{v}_{n} d S=1$. Hence, for $N=1$, it is clear that $\boldsymbol{v}$ obeys Eq. (12) for $\boldsymbol{f}=0$ and therefore Eq. (14) does not have a unique solution if any. It is possible to draw similar conclusions for $N \geq 2$ with this time $\boldsymbol{v}$ solution of Eq. (12) for $\boldsymbol{f}=0$. Consequently, the right-hand side of Eq. (14) must satisfy compatibility conditions for Eq. (14) to have at least one solution! To specify those conditions, we first recall (see Pozrikidis ${ }^{20}$ ) that for arbitrary surfaces $S_{n}$ and $S_{m}$ (with either $m=n$ or $m \neq n$ )

$$
\int_{S_{0}} n_{k}\left(\boldsymbol{x}_{0}\right) T_{i j k}\left(\boldsymbol{x}, \boldsymbol{x}_{0}\right) d S\left(\boldsymbol{x}_{0}\right)=0,
$$

for $\boldsymbol{x}$ on $S_{n}$ and

$$
\int_{S_{m}} n_{k}\left(\boldsymbol{x}_{0}\right) T_{i j k}\left(\boldsymbol{x}, \boldsymbol{x}_{0}\right) d S\left(\boldsymbol{x}_{0}\right)=4 \pi \delta_{i j} \delta_{m n},
$$

for $\boldsymbol{x}$ on $S_{n}$ (when $m=n$, the integral (18) is a weakly singular integral in the principal value sense of Cauchy).

Multiplying Eq. (14) by the normal vector $\boldsymbol{n}\left(\boldsymbol{x}_{0}\right)$ and integrating over the surface $S_{n}$, one thus arrives at the following compatibility relations for the right-hand side $S\left(x_{0}\right)$ of Eq. (14):

$$
\int_{S_{n}} \boldsymbol{S}\left(\boldsymbol{x}_{0}\right) \cdot \boldsymbol{n}\left(\boldsymbol{x}_{0}\right) d S\left(\boldsymbol{x}_{0}\right)=0,
$$

for $n=1, \ldots, N$. Noting that $G_{i j}\left(\boldsymbol{x}, \boldsymbol{x}_{0}\right)=G_{j i}\left(\boldsymbol{x}_{0}, \boldsymbol{x}\right)$, exploiting the identities (13) and the definition (15) of the right-hand side $S$ immediately shows that the above conditions (19) are satisfied for the addressed boundary-integral equation (14). Accordingly, Eq. (14) has solutions $\boldsymbol{u}$, which read $\boldsymbol{u}=\boldsymbol{u}_{0}$ $+\boldsymbol{v}_{\beta_{1}, \ldots, \beta_{N}}$ with $\boldsymbol{u}_{0}$ any solution. For the present work, we require the selected solution $\boldsymbol{u}$ to comply with the relations (7). This is achieved by using Wielandt's deflation technique as performed, among others, by Loewenberg and Hinch, ${ }^{25}$ Zinchenko et al., ${ }^{26}$ and explained in details in Kim and Karrila. ${ }^{21}$ For $N \geq 1$ bubbles, it is then possible to prove that $\boldsymbol{u}$ solution to Eqs. (14) and (7) obeys the modified, well-posed, and coupled boundary-integral equations

$$
\begin{aligned}
\boldsymbol{u}\left(\boldsymbol{x}_{0}\right) & -\frac{1}{4 \pi} f_{S} \boldsymbol{u}(\boldsymbol{x}) \cdot \boldsymbol{T}\left(\boldsymbol{x}, \boldsymbol{x}_{0}\right) \cdot \boldsymbol{n}(\boldsymbol{x}) d S(\boldsymbol{x}) \\
- & \sum_{n=1}^{N}\left[\frac{\int_{S_{n}} \boldsymbol{u}(\boldsymbol{x}) \cdot \boldsymbol{n}(\boldsymbol{x}) d S(\boldsymbol{x})}{\int_{S_{n}} d S(\boldsymbol{x})}\right] \boldsymbol{n}\left(\boldsymbol{x}_{0}\right)=\boldsymbol{S}\left(\boldsymbol{x}_{0}\right),
\end{aligned}
$$

for $\boldsymbol{x}_{0}$ on $S_{n}, n=1, \ldots, N$.

Clearly, $\boldsymbol{u}$ solution to Eqs. (14) and (7) obeys Eq. (20). Conversely, multiplying Eq. (20) by $\boldsymbol{n}\left(\boldsymbol{x}_{0}\right)$ and integrating over the surface $S_{n}$ this time shows that, using Eqs. (17), (18), and (19), a solution $\boldsymbol{u}$ to Eq. (20) also fulfils the relations (7) and thus obeys Eq. (14). Hence, the unique and required solution to both Eqs. (14) and (7) is the solution of the coupled boundary-integral equations (20), which is therefore well-posed. Moreover, as the reader may easily check, the homogeneous counterpart of Eq. (20), here obtained by selecting $\boldsymbol{S}=\mathbf{0}$, has spectrum $[-1,1]$.

\section{Case of the axisymmetric fluid domain}

The entire material developed in Secs. III A and III B is actually valid for arbitrary three-dimensional $\mathrm{N}$-bubble clusters. However, as previously mentioned in the Introduction, the numerical counterpart results in heavy implementation step and computations. Therefore, we confine the analysis to axisymmetric configurations, as the one depicted in Fig. 1, and non-axisymmetric instabilities are thus not treated here. For convenience, we further adopt cylindrical coordinates $(r, \phi, z)$ with $r=\sqrt{x^{2}+y^{2}}$ and $\phi$ the azimuthal angle in the range $[0,2 \pi]$. In Eq. (14), we perform the integration in the azimuthal direction. Setting $\boldsymbol{u}=u_{r} \boldsymbol{e}_{r}+u_{z} \boldsymbol{e}_{z}=u_{\alpha} \mathbf{e}_{\alpha}$ (with $\alpha=r, z)$ in the liquid and $\boldsymbol{f}=f_{r} \boldsymbol{e}_{r}+f_{z} \boldsymbol{e}_{z}=f_{\alpha} \boldsymbol{e}_{\alpha}$ on the liquid boundary then makes it possible to cast Eq. (14) into the following form: 


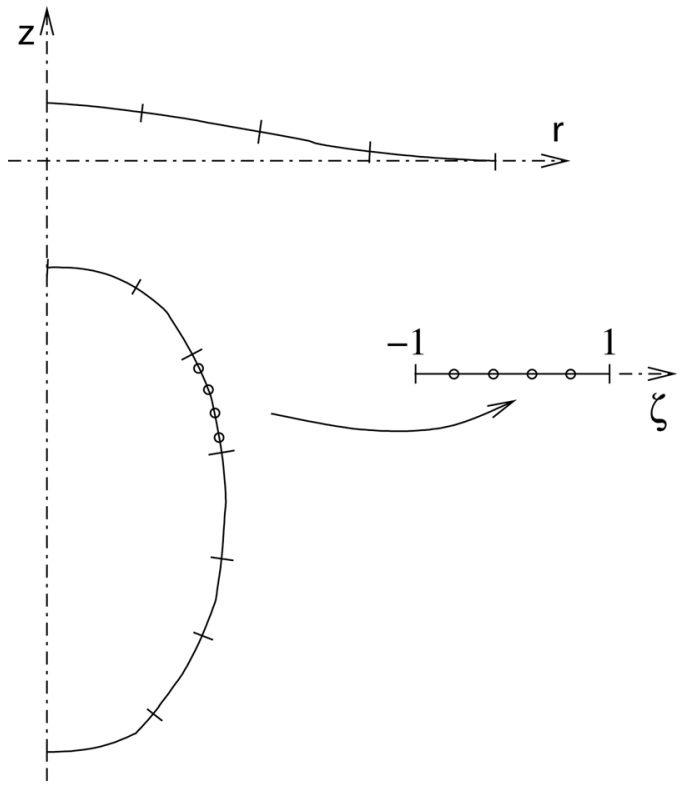

FIG. 2. Discretization and mapping of each element onto $[-1,1]$ with end points and collocation points are indicated by small segments or circles, respectively.

$$
\begin{aligned}
4 \pi u_{\alpha}\left(\boldsymbol{x}_{0}\right)-f_{\mathcal{L}} C_{\alpha \beta}\left(\boldsymbol{x}, \boldsymbol{x}_{0}\right) u_{\beta}(\boldsymbol{x}) d l(\boldsymbol{x}) \\
=-\frac{1}{\mu} \int_{\mathcal{L}} B_{\alpha \beta}\left(\boldsymbol{x}, \boldsymbol{x}_{0}\right)\left(\gamma \nabla_{S} \cdot \boldsymbol{n}-\rho g z\right) n_{\beta}(\boldsymbol{x}) d l(\boldsymbol{x}),
\end{aligned}
$$

where $\mathcal{L}_{n}$ is the trace of the surface $S_{n}$ and $\mathcal{L}=\cup_{m=0}^{N} \mathcal{L}_{n}$ the entire contour. The quantity $d l$ in the $\phi=0$ half-plane (see also Fig. 2) is the differential arc length. The resulting $2 \times 2$ square matrices $B_{\alpha \beta}\left(\boldsymbol{x}, \boldsymbol{x}_{0}\right)$ and $C_{\alpha \beta}\left(\boldsymbol{x}, \boldsymbol{x}_{0}\right)$, called single-layer and double-layer and available in Pozrikidis, ${ }^{20}$ are written in Appendix A.

\section{NUMERICAL IMPLEMENTATION}

The boundary-integral equation (14) and the relations (7) are solved using a collocation method and a discrete Wielandt's deflation technique. The implemented steps are described below.

\section{A. Employed boundary elements and discretized boundary-integral equation}

Following Muldowney and Higdon's, ${ }^{27}$ the liquid contour $\mathcal{L}=\cup_{m=0}^{N} \mathcal{L}_{n}$ is divided into $N_{e}$ curved boundary elements arranged to preserve the $x \rightarrow-x$ symmetry. Each element has two end points, and $N_{c}$ collocation points spread by a uniform or Gauss distribution. As seen in Fig. 2, the end point of an element can (i) belong to two elements located off the axis of symmetry, or (ii) either be on the symmetry axis or (iii) be completely free at the tip of the truncated contour $\mathcal{L}_{0}$ modeling the free surface.

Isoparametric interpolations are employed for the location of a point $\boldsymbol{x}^{i_{e}}$ belonging to the boundary element $i_{e}$ and for the approximation of the associated velocity $\boldsymbol{u}\left(\boldsymbol{x}^{i_{e}}\right)$ and surface traction $\boldsymbol{f}\left(\boldsymbol{x}^{i_{e}}\right)$ with

$\boldsymbol{x}^{i_{e}}=\sum_{i_{c}=1}^{N_{c}} L_{i_{c}}(\zeta) \boldsymbol{x}_{i_{c}}^{i_{e}}, \boldsymbol{u}\left(\boldsymbol{x}^{i_{e}}\right)=\sum_{i_{c}=1}^{N_{c}} L_{i_{c}}(\zeta) \boldsymbol{u}_{i_{c}}^{i_{e}}, \boldsymbol{f}\left(\boldsymbol{x}^{i_{e}}\right)=\sum_{i_{c}=1}^{N_{c}} L_{i_{c}}(\zeta) \boldsymbol{f}_{i_{c}}^{i_{e}}$, where $L_{i_{c}}$ designates the employed $\left(N_{c}-1\right)$-order Lagrangian interpolant polynomial and $\zeta$ the variable on the segment $[-1,1]$ onto which each boundary element is mapped. The quantities $\boldsymbol{n} \cdot \boldsymbol{e}_{z}, \boldsymbol{n} \cdot \boldsymbol{e}_{r}$ and $\nabla_{S} \cdot \boldsymbol{n}$ are also expressed at the point $\boldsymbol{x}^{i_{e}}$ in Appendix B. Collecting at our $N_{e} N_{c}$ nodal points the components $\left(\rho \boldsymbol{g} \cdot \boldsymbol{x}+\gamma \nabla_{S} \cdot \boldsymbol{n}\right) \boldsymbol{n} \cdot \boldsymbol{e}_{\alpha}$ and $\boldsymbol{u} \cdot \boldsymbol{e}_{\alpha}$ for $\alpha=r, z$ in prescribed and unknown $2 N_{e} N_{c}$ vectors $\boldsymbol{F}$ and $\boldsymbol{U}$ and exploiting Eq. (22) makes it possible to cast the boundaryintegral equation (21) into the $2 N_{e} N_{c}$-equation linear system

$$
\boldsymbol{U}-\boldsymbol{C} \cdot \boldsymbol{U}=\boldsymbol{B} \cdot \boldsymbol{F} .
$$

Matrices $\boldsymbol{B}$ and $\boldsymbol{C}$ are given in Appendix B as integrals over the segment $[-1,1]$ involving the quantities $B_{\alpha \beta}\left(\boldsymbol{x}, \boldsymbol{x}_{0}\right)$ and $C_{\alpha \beta}\left(\boldsymbol{x}, \boldsymbol{x}_{0}\right)$ defined in Appendix $\mathrm{A}$ and are here accurately computed, by exploiting the polynomial approximations given in Abramowitz and Stegun, ${ }^{28}$ of the complete elliptic integrals of the first and second kind (see also ${ }^{20}$ )

$$
\begin{aligned}
& F(k)=\int_{0}^{\pi / 2} \frac{d \phi}{\left(1-k^{2} \cos ^{2} \phi\right)^{1 / 2}}, \\
& E(k)=\int_{0}^{\pi / 2}\left(1-k^{2} \cos ^{2} \phi\right)^{1 / 2} d \phi .
\end{aligned}
$$

One should note that the velocity $\boldsymbol{u}$ is unknown at each nodal point of the truncated and discretized free-surface contour $\mathcal{L}_{0}$. Therefore, the tips of this truncated free-surface are not fixed in the numerical computations.

\section{B. Computation of the matrices $B$ and $C$}

The components of the matrices $\boldsymbol{B}$ and $\boldsymbol{C}$ are reduced if necessary to regular integrals. They are accurately computed as explained in Appendix B by employing the self-adaptative method proposed by Voutsinas and Bergeles. ${ }^{29}$ Here, we iteratively divide a segment $[a, b]$ into equal or inequal subsegments. The encountered regular integral over each subsegment is calculated by employing classical Gauss quadratures. The iterative procedure is stopped as soon as the computed value of the integral reaches a prescribed relative accuracy between $10^{-3}$ to $10^{-4}$ (obtained in practice using three or four iterations).

\section{Discrete Wielandt's deflation}

As previously explained in Sec. III B, the linear system (23) involves the matrix $\boldsymbol{C}$ with a discrete spectrum having a finite number of eigenvalues in the interval $[-1,1]$ and the eigenvalues close to unity prevent one to accurately invert Eq. (23). Here we remove the eigenvalues of $\boldsymbol{C}$ close to unity without affecting the other eigenvalues by implementing a so-called Wielandt's deflation. ${ }^{21}$ We compute the (discrete) spectrum of the $\boldsymbol{C}$ matrix by the $Q R$ method, ${ }^{30}$ select $n_{\lambda_{1}}$ eigenvalues close to unity with associated eigenvector $\mathbf{V}_{n}$ for the adjoint with eigenvalue $\lambda_{n}$ and introduce the new matrix $\boldsymbol{C}^{\prime}$ as

$$
\boldsymbol{C}^{\prime}=\boldsymbol{C}-\sum_{n=1}^{n_{\lambda_{1}}} \lambda_{n} \boldsymbol{Z}_{n} \otimes \boldsymbol{V}_{n}, \quad \boldsymbol{Z}_{n}=\boldsymbol{V}_{n} /\left\|\boldsymbol{V}_{n}\right\|^{2},
$$

with $\left\|\boldsymbol{V}_{n}\right\|$ the discrete norm of the vector $\boldsymbol{V}_{n}$ calculated using the entire discretized surface $S=\cup_{m=0}^{N} S_{n}$. Since the 
eigenvector $\boldsymbol{V}_{n}$ must be collinear on $\boldsymbol{S}$ to the normal vector $\boldsymbol{n}$, the scalar product of $\boldsymbol{V}_{n} \cdot(\boldsymbol{B} \cdot \boldsymbol{F})$ must be practically equal to zero and therefore the linear system (23) is replaced with the well-posed one

$$
\boldsymbol{U}-\boldsymbol{C}^{\prime} \cdot \boldsymbol{U}=\boldsymbol{B} \cdot \boldsymbol{F},
$$

which is solved by $L U$ decomposition. ${ }^{31}$

\section{Fluid interfaces tracking}

Each surface's shape is tracked in time by exploiting the conditions (6). In practice, the knowledge of the fluid velocity at each collocation point at time $t$ is used to move between times $t$ and $t+d t$ the position of each nodal point $\boldsymbol{x}_{i_{c}}^{i_{e}}$ by integrating the equation

$$
\frac{d \boldsymbol{x}_{i_{c}}^{i_{e}}(t)}{d t}=\boldsymbol{u}_{i_{c}}^{i_{e}}(t)
$$

This is numerically achieved by using a Runge-Kutta-Fehlberg method. The time step is adapted by controlling the numerical error between the computations at a second and a third Runge-Kutta algorithms. The new time step is determined following the relationship

$$
d t_{\text {new }}=d t \sqrt[3]{3 \frac{\varepsilon}{\left\|\overline{\boldsymbol{x}}_{i_{c}}^{i_{e}}(t+d t)-\hat{\boldsymbol{x}}_{i_{c}}^{i_{e}}(t+d t)\right\|}}
$$

where $^{32} \varepsilon>0$ is a predefined accuracy, $\overline{\boldsymbol{x}}_{i_{c}}^{i_{e}}(t+d t)$ and $\hat{\boldsymbol{x}}_{i_{c}}^{i_{e}}(t+d t)$ are the computed locations at the second and third orders, respectively. The set of coefficients required in the Runge-Kutta algorithms are taken from the book of Stoer and Bulirsch. ${ }^{30}$

As time evolves, collocation points have been seen to concentrate near a stagnation area (a bubble rear) of the bubble(s), therefore, yielding stretched and thus unsuitable mesh(es) for the bubble(s). Such issues are circumvented by redistributing from time to time the collocation points. In addition, the typical length of the boundary elements is adequately reduced in the area where two interfaces are close by distributing elements nonuniformly, following a geometric sequence.

\section{NUMERICAL RESULTS}

This section presents and discusses our numerical results for a few suitable benchmark tests and for the timedependent shapes of the free surface and one, two, or three bubble(s). We study bubble-surface and bubble-bubble interactions act, and the competition between such interactions.

\section{A. Benchmark tests}

Three tests have been performed for one bubble.

\section{Integral identities}

According to (13), (17), and (18) and the symmetries of the stress tensor $\boldsymbol{T}$ (recall (11)), the following identities hold for arbitrary point $x_{0}$ located on the bubble surface $S$

$$
\begin{gathered}
\int_{S} G_{i j}\left(\boldsymbol{x}, \boldsymbol{x}_{0}\right) n_{i}(\boldsymbol{x}) d S(\boldsymbol{x})=0 \\
\int_{S} T_{i j k}\left(\boldsymbol{x}, \boldsymbol{x}_{0}\right) n_{k}(\boldsymbol{x}) d S(\boldsymbol{x})=-4 \pi \delta_{i j} .
\end{gathered}
$$

In the axisymmetric formulation, the bubble has an associated contour $\mathcal{L}$ (the trace of its surface $S$ in the $\phi=0$ halfplane) and the previous relations then become

$$
I_{\alpha}^{s}=\int_{\mathcal{L}} B_{\alpha \beta}\left(\boldsymbol{x}, \boldsymbol{x}_{0}\right) n_{\beta}(\boldsymbol{x}) d l(\boldsymbol{x})=0,
$$

for $\alpha=r, z$, and

$$
\begin{gathered}
I_{z z}^{d}=\int_{\mathcal{L}} C_{z z}\left(\boldsymbol{x}, \boldsymbol{x}_{0}\right) d l(\boldsymbol{x})=-4 \pi, \\
I_{r z}^{d}=\int_{\mathcal{L}} C_{r z}\left(\boldsymbol{x}, \boldsymbol{x}_{0}\right) d l(\boldsymbol{x})=0 .
\end{gathered}
$$

Indeed, the introduction of the velocity and unit normal in the polar reference frame leads to a composition of the Cartesian components $T_{i j k}$. Using the definition of $\boldsymbol{C}$ easily shows that (30) yields only relations (32) and (33) for the components $C_{z z}$ and $C_{r z}$. By contrast to the tridimensional formulation, ${ }^{14}$ it is therefore not possible to regularize the double-layer potential in Eq. (21) by solely using the identities (32) and (33).

The computed average (over all collocation points) of the absolute value of the "single-layer" integral $I_{\alpha}^{S}$ arising in Eq. (31) is displayed in Table I using $N_{e}=1,4$, and 16

\begin{tabular}{|c|c|c|c|c|c|c|c|c|c|}
\hline & & \multicolumn{3}{|c|}{ (a) $I_{r}^{S}$} & & & \multicolumn{3}{|c|}{ (b) $I_{z}^{s}$} \\
\hline & & \multicolumn{3}{|c|}{$N_{g}$} & & & \multicolumn{3}{|c|}{$N_{g}$} \\
\hline & & 4 & 6 & 8 & & & 4 & 6 & 8 \\
\hline \multirow[t]{3}{*}{$N_{e}$} & 1 & $4.8 \cdot 10^{-2}$ & $1.9 \cdot 10^{-2}$ & $5.6 \cdot 10^{-3}$ & \multirow[t]{3}{*}{$N_{e}$} & 1 & $5.7 \cdot 10^{-2}$ & $2 \cdot 10^{-2}$ & $4.6 \cdot 10^{-3}$ \\
\hline & 4 & $2.3 \cdot 10^{-4}$ & $1.3 \cdot 10^{-5}$ & $1.5 \cdot 10^{-5}$ & & 4 & $2.1 \cdot 10^{-4}$ & $4.1 \cdot 10^{-6}$ & $5.6 \cdot 10^{-6}$ \\
\hline & 16 & $2.6 \cdot 10^{-6}$ & $1.3 \cdot 10^{-6}$ & $3.6 \cdot 10^{-6}$ & & 16 & $6 \cdot 10^{-7}$ & $2.9 \cdot 10^{-7}$ & $7.1 \cdot 10^{-7}$ \\
\hline
\end{tabular}
boundary elements on a sphere with unit diameter, $N_{c}=4,6$, and 8 collocation points on each element and 8 Gauss points to compute integrals over each partition.

Clearly, a good convergence is observed with, for $N_{e}$ and $N_{c}$ large enough, a $O\left(10^{-7}\right)$ error comparable with the

TABLE I. Average of the absolute value of the integral (a) $I_{r}^{s}$ and (b) $I_{z}^{s}$ versus the numbers $N_{e}$ and $N_{c}$ of boundary elements and collocation points on each element, respectively. 
TABLE II. Average of the absolute value of the quantity (a) $I_{z z}^{d} / 4 \pi-1$ and (b) integral $I_{r z}^{d}$ versus the numbers $N_{e}$ and $N_{c}$ of boundary elements and collocation points on each element.

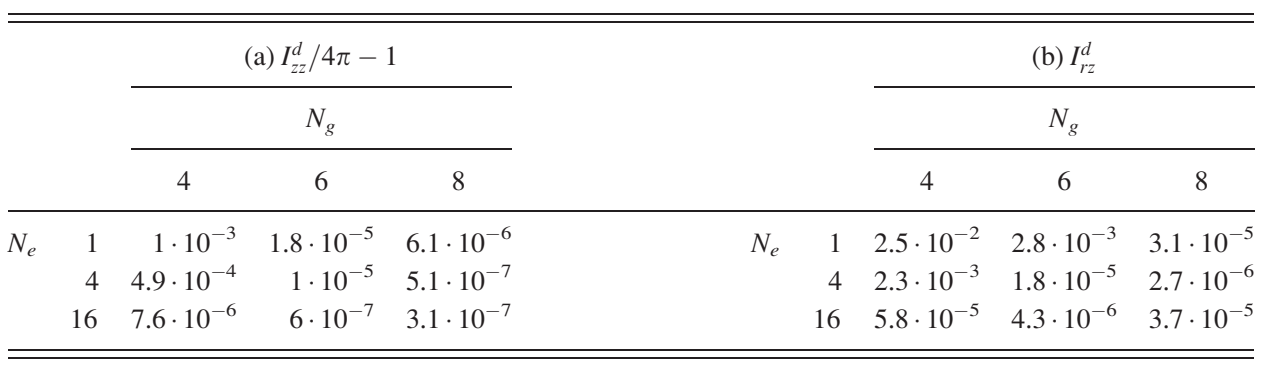

obtained accuracy in computing the elliptic integrals of first and second kind given, respectively, by Eq. (24) using Abramowitz and Stegun. ${ }^{28}$

Similar results for the "double-layer" average values of the quantities $I_{z z}^{d} / 4 \pi-1$ and $I_{r z}^{d}$ are given in Table II for the same bubble and values of the integers $N_{e}$ and $N_{c}$. Again, a very good agreement with the theory is found.

\section{Ascending bubble}

As pointed out by Taylor and Acrivos ${ }^{33}$ and Pan and Acrivos, ${ }^{34}$ within our assumption of negligible inertial effects, a bubble immersed in an unbounded liquid having uniform surface tension $\gamma$ and spherical shape with radius $a$ at initial time remains spherical with radius $a$ when ascending under the action of a uniform gravity $\boldsymbol{g}=-g \boldsymbol{e}_{z}$. The bubble translates at the velocity $\boldsymbol{u}=U \boldsymbol{e}_{z}$ with $U=\rho g a^{2} /(3 \mu)$ whatever the Bond number $\mathrm{Bo}=\rho g a^{2} /(3 \gamma)$ and at its surface the velocity components $u_{r}$ and $u_{z}$ read (this is the so-called Hadamard-Rybczynski solution ${ }^{35,36}$ )

$$
\begin{gathered}
u_{r}=U \frac{\sin 2 \theta}{4}, \\
u_{z}=U\left(1-\frac{\sin ^{2} \theta}{2}\right),
\end{gathered}
$$

with $\theta$ the angle between the vector $\boldsymbol{e}_{z}$ and the radial direction. Computed values of $u_{r} / U$ and $u_{z} / U$ for $\mathrm{Bo}=1000$ are compared against the analytical solutions (34)-(35) in Figs. 3 and 4.

Numerical results perfectly match the analytical ones even with the coarsest grid. Actually, the computed average relative error is order $0.1 \%$ when $N_{e}=4$ and becomes order $10^{-3} \%$ for refined meshes $N_{e}=8,20$.

\section{Discrete spectrum of the discretized operator C}

As mentioned in Sec. IV, a key step in accurately inverting the discretized system (23) is the computation of the eigenvalues $\lambda$ of the linear operator $\boldsymbol{C}$. First, we consider a spherical bubble distant from the free surface. Using Lamb's solutions, Kim and Karrila ${ }^{21}$ theoretically predicted these values to be

$$
\begin{aligned}
& \lambda_{n}^{-}=\frac{-3}{(2 n-1)(2 n+1)}, \quad n=1,2, \ldots, \\
& \lambda_{n}^{+}=\frac{3}{(2 n+1)(2 n+3)}, \quad n=0,1, \ldots
\end{aligned}
$$

The computed values are compared with Eqs. (36)-(37) in Fig. 5 for different meshes of the bubble contour. For a given mesh, there is only one eigenvalue close to unity.

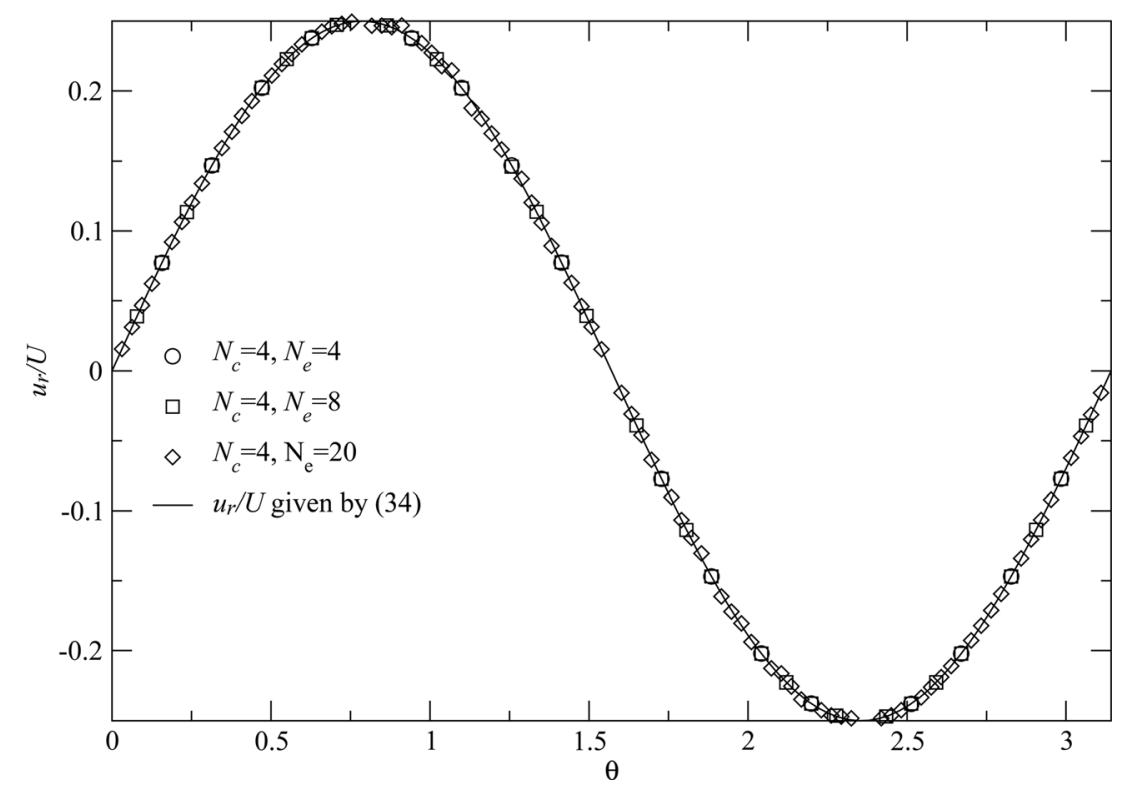

FIG. 3. Normalized velocity $u_{r} / U$ velocity versus $\theta$ computed for $\mathrm{Bo}=10^{3} ; N_{e}=4,8,20$; and $N_{c}=4$. The analytical solution (34) is given by the solid line. 


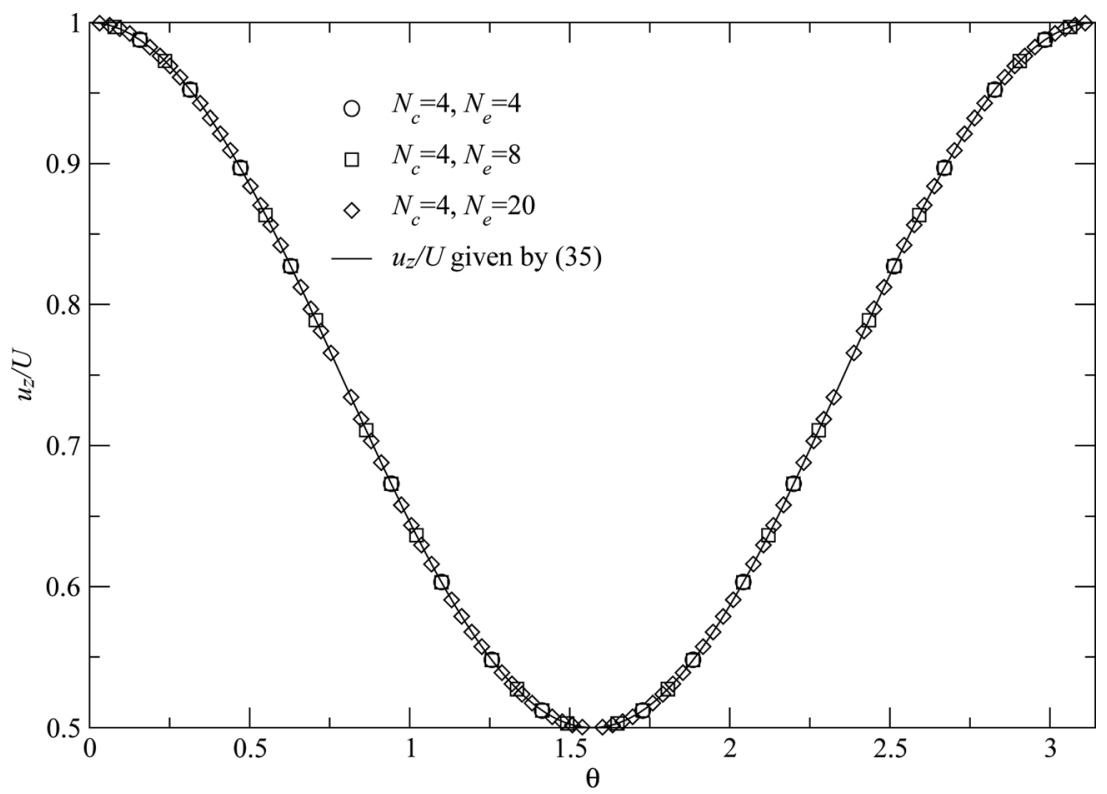

FIG. 4. Normalized velocity $u_{z} / U$ velocity versus $\theta$ computed for Bo $=10^{3} ; N_{e}=4,8,20$; and $N_{c}=4$. The analytical solution (35) is given by the solid line.

When the bubble approaches a free surface for a similar number of collocation points, there is a similar number of discrete eigenvalues, but these values tend to concentrate near the end point -1 and +1 as the gap between the free surface and the bubble decreases. This trend is clearly observed in Fig. 6 both for undeformed and deformed liquid surfaces. In such circumstances, one needs to apply Wielandt's deflation to all eigenvalues located close to unity.

\section{B. Results for one bubble}

Here, we consider the motion of one bubble toward a fluid interface under buoyancy effects. As mentioned in the Introduction, this case is encountered in various applications, such as in glass melting. For instance, the axisymmetric film drainage between a droplet and a free surface has been studied by Chi and Leal, ${ }^{10}$ using a boundary integral formulation. However, Chi and Leal confined the investigations to droplets with a non-zero viscosity ratio between fluid inside the droplet and fluid outside. In the present work, the case of bubbles rising toward a fluid interface can be studied.

In practice, it is easier to work under dimensionless form. At initial time, the bubble is spherical with radius $a$. We henceforth take, respectively, $2 a, U=\rho g a^{2} /(3 \mu)$, and $2 a / U=6 \nu /(g a)$ as length, velocity, and time scales. The normalized surface traction $f$ using Eq. (5) and setting $p_{1}=0$ in the bubble then reads

$$
\boldsymbol{f}=\left(\frac{\nabla_{S} \cdot \boldsymbol{n}}{\text { Во }}-12 z\right) \boldsymbol{n},
$$

with Bo, the Bond number, defined as

$$
\mathrm{Bo}=\frac{\rho g a^{2}}{3 \gamma} .
$$

Here, we concentrate on the film drainage between the bubble and the free surface, which takes place after a pure rising regime of the bubble. Of course, both bubble and free

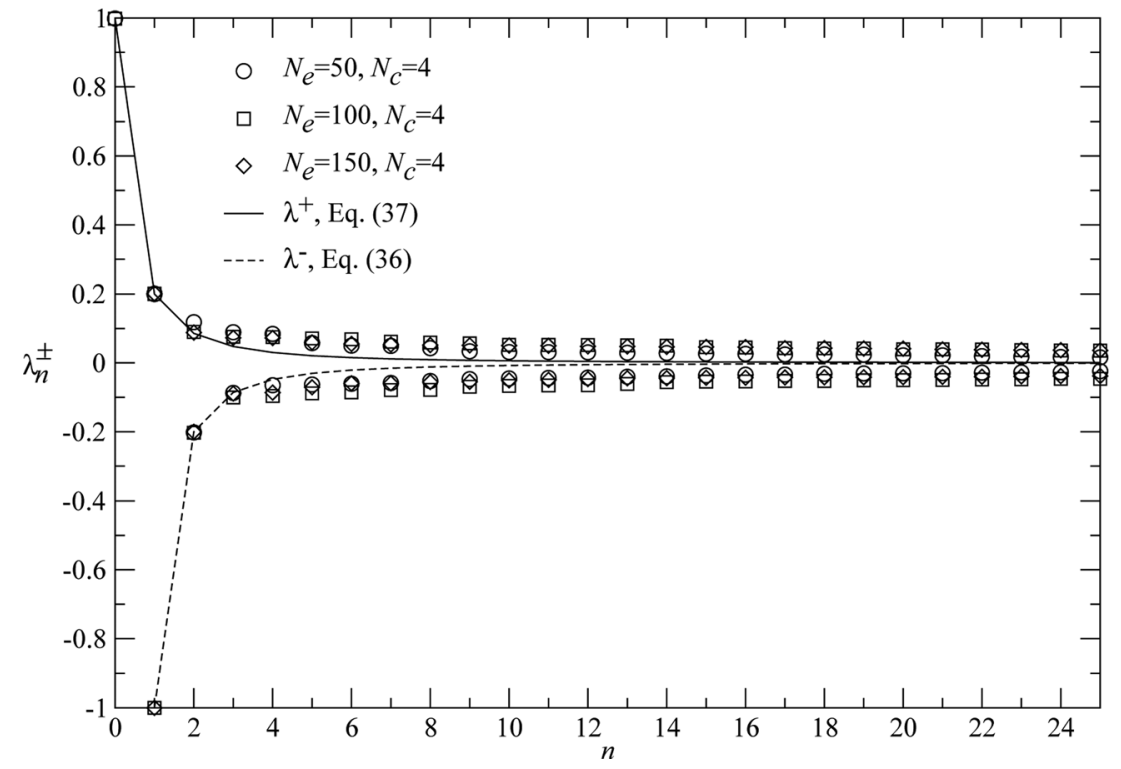

FIG. 5. Computed eigenvalues and analytical predictions (36)-(37) for a spherical bubble immersed in an unbounded liquid. 


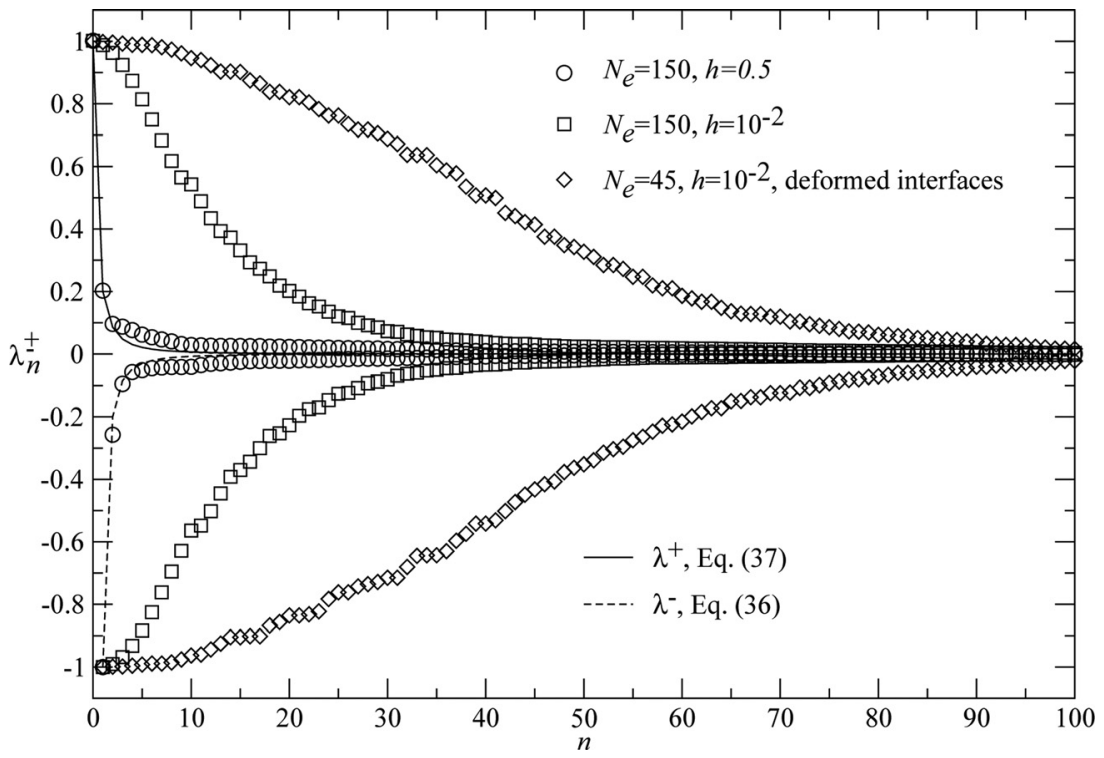

FIG. 6. Computed eigenvalues for a spherical or nonspherical bubble close to a free surface and comparisons with Eqs. (36)-(37) for a spherical bubble immersed in an unbounded liquid.

surface interfaces are likely to depend upon the initial location and shape of the bubble. In our numerical procedure, the free surface is moreover truncated and we therefore carefully investigate to which extent both the initial location of the bubble and the size of the truncated free surface affect the results.

Henceforth, the film thickness $h$ designates the gap (normalized by $2 a$ ) on the $z$-axis between the bubble surface and the free surface (note that $h=h_{1} /(2 a)$ with $h_{1}$ shown in Fig. 1).

The bubble and free surface interfaces are tracked as explained in Sec. IV, using a self-adapted time step. More precisely, when the bubble is far from the interface, the time step is large and nearly constant between two time iterations. In contrast, when the bubble is very close to the free surface, the prescribed accuracy requires to decrease the time step. In practice, numerical computations are stopped as soon as the film thickness reaches a value of order $10^{-2}$, or whenever the time step suitable to guarantee a prescribed accuracy becomes too small.

\section{Sensitivity to the domain truncation}

Numerical simulations ${ }^{37}$ have been achieved at $\mathrm{Bo}=10$, with initial gap between the spherical bubble and the flat (undeformed) interface equal to $1 / 2$. The effect of the domain truncation has been first investigated by running simulations for a fluid interface extending over 5 and 10 bubble diameters using, respectively, 20 and 25 boundary elements on the bubble and on the free surface interfaces. The number $N_{c}$ of collocation points (see Sec. IV) is equal to 4 .

Fig. 7 presents for these numerical simulations both the film thickness $h$ and the relative error $\Delta \mathcal{V}(t)=\mathcal{V}(t) / \mathcal{V}(0)-1$ for the preserved bubble volume $\mathcal{V}(t)$, see Eq. (7).

Starting from a value equal to $0.5, h$ is seen to rapidly decrease at small time when the bubble is free to rise. For $t \gtrsim \mathcal{O}(1)$, the film thickness $h$ slowly drops due to the drainage of the liquid between the very close interfaces of the bubble and of the free surface. The two numerical investigations clearly yield very close results. Therefore, truncating the free surface at a distance exceeding five bubble diameters appears to be sufficient.

\section{Sensitivity to the bubble initial location}

This time, numerical simulations are performed with two different initial locations of the bubble below the fluid interface, whereas the free surface is truncated at 5 bubble

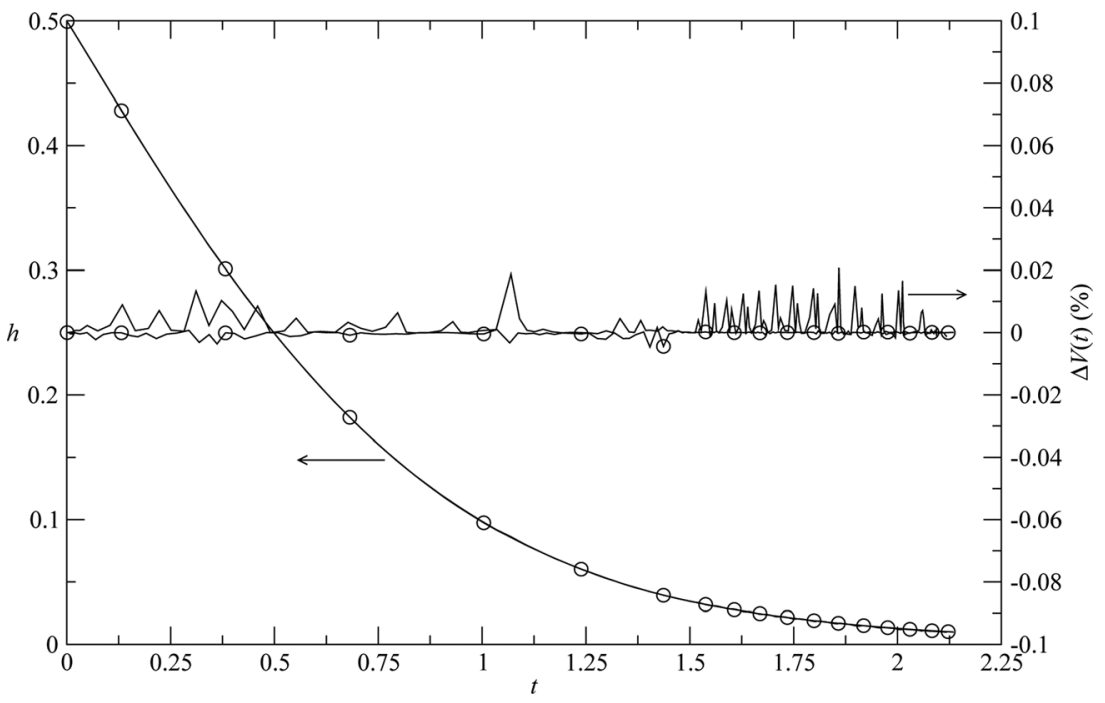

FIG. 7. Film thickness $h$ and relative error of $\Delta \mathcal{V}(t)$ for the bubble volume as a function of time for a fluid interface truncated at a distance equal to 10 bubble diameters $(-)$ and equal to 5 bubble diameters $(\circ-0)$. Here, $\mathrm{Bo}=10$ and the initial distance between the spherical bubble and the flat interface is $1 / 2$. 


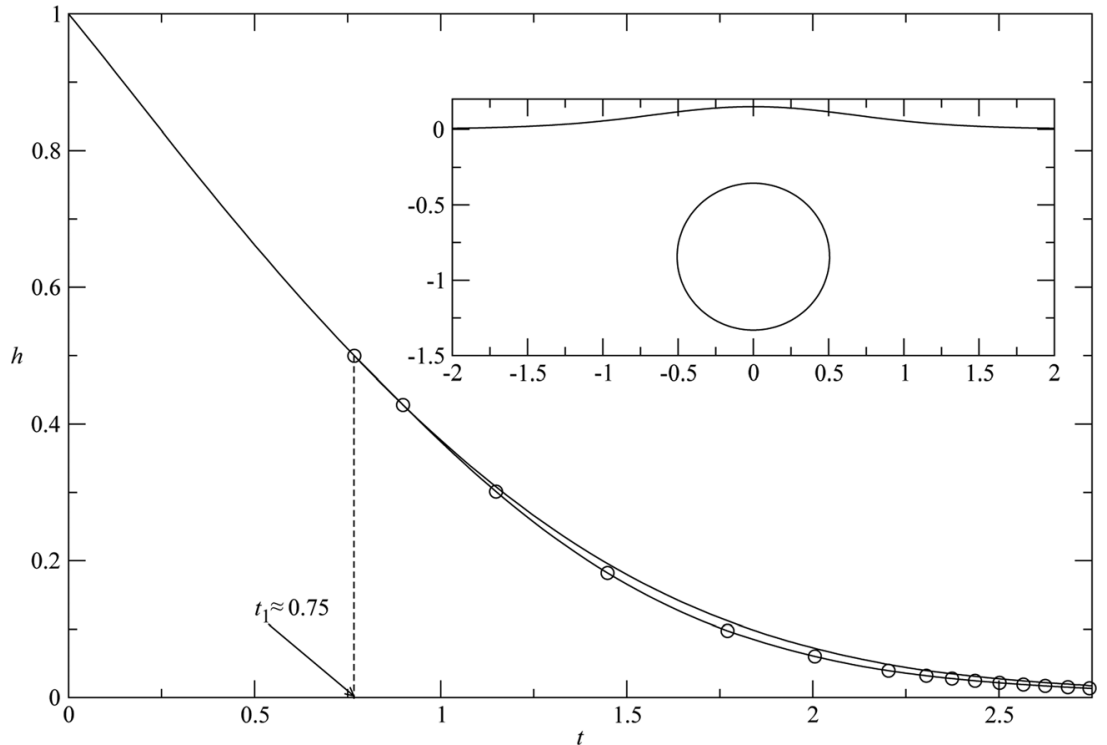

FIG. 8. Film thickness $h$ versus time $t$ for a bubble located initially at $h(0)=1$ (一) and for a bubble located initially at $h(0)=1 / 2(\circ-\circ)$ with $\mathrm{Bo}=10$. The distant bubble is located at $h=1 / 2$ at $t_{1} \approx 0.75$ as depicted in the inset.

diameters. The numbers of boundary elements are identical to the previous calculations and again $N_{c}=4$ and $\mathrm{Bo}=10$.

Fig. 8 plots $h$ versus time $t$ for two bubbles: a distant one ( $h=1$ at $t=0$ ) and another one starting from $h=1 / 2$. More precisely, the distant bubble rises with $h=1 / 2$ at time $t_{1} \approx 0.75$, at which we let the other bubble start its motion (as illustrated in Fig. 8). The first stage of the bubble started from $h=1 / 2$ with undeformed interfaces is slightly different from the case where the bubble is initially located at $h=1$. Nevertheless, after the rising step, the two curves present the same trend.

The numerical value $t_{1} \approx 0.75$ at which the distant bubble starting at $h=1$ arrives at $h=1 / 2$ is in good agreement with the typical rising velocity of a bubble. Indeed, as shown in Sec. V B 4, $h$ obeys Eq. (41) for non-deformable interfaces. Solving Eq. (41) for weak hydrodynamic interactions then shows that the time to reduce $h$ of $1 / 2$ is $3 / 4$, which is very close to $t_{1}$.

In view of the previous results, computations are henceforth achieved with an initial bubble-interface gap of one bubble radius and by truncating the interface beyond 5 bubble diameters.

\section{Bubble shape's sensitivity to the Bond number Bo}

As already pointed out, two different steps are observed in the bubble motion. The first one is the free rising of the bubble, which depends on its hydrodynamic interactions with the fluid interface. The second one is the key drainage step, in which the bubble is nearly at rest. In this second stage, the bubble's shape is driven by the competition between the buoyancy and surface tension forces. Consequently, the bubble's shape is a function of the Bond number. This shape has been determined by Princen ${ }^{3}$ for a drop very close to the fluid interface by requiring the hydrostatic pressure balance.

Fig. 9 presents bubble's shapes when the bubble is nearly at rest for Bond numbers equal to $0.1,1$, and 10. Bubble's shapes predicted by the Princen's model are also drawn in Fig. 9 using dashed lines.

For the weak value of the Bond number, Bo $=0.1$, gravity effects are small compared with surface tension effects, and as seen in Fig. 9(a), the bubble remains nearly spherical when rising and the free surface is weakly deformed. By contrast, as Bo increases both the free surface and the bubble interface are affected and the bubble shape becomes nonspherical and evolves from a lens at $\mathrm{Bo}=1$, see Fig. 9(b) to a quasi-hemispherical form at $\mathrm{Bo}=10$, Fig. 9(c). In any case, the bubble however keeps its volume constant as retrieved by the numerics (see the given relative error volume given in Fig. 9 caption).

The area where the drainage is at play increases with the Bond number, and shrinks as the Bond number decreases. It tends to zero when Bo $=0$. A plateau is observed for the drainage area at very large Bo. From this variety of shapes of bubbles, we can expect to see the influence of the Bond number on the film drainage. (a) $\mathrm{Bo}=0.1$

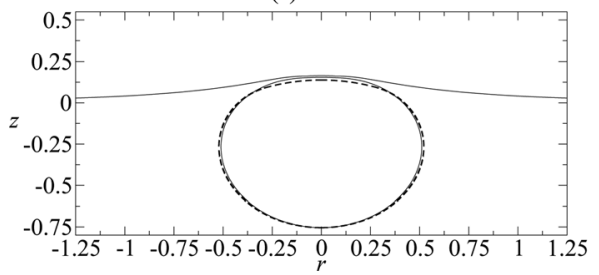

(b) $\mathrm{Bo}=1$

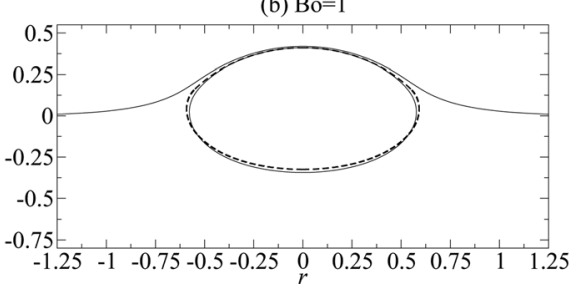

(c) $\mathrm{Bo}=10$

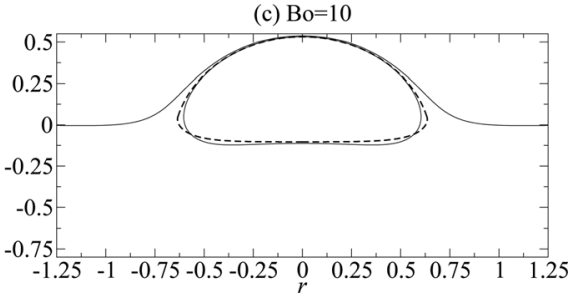

FIG. 9. Bubble shape close to the free surface at (a) Bo=0.1 with $\Delta \mathcal{V}=-1.1 \cdot 10^{-3} \%$, (b) Bo=1 with $\Delta \mathcal{V}=1.7 \cdot 10^{-4} \%$ and (c) Bo $=10$ with $\Delta \mathcal{V}=2.4 \cdot 10^{-5} \%$. Dashed lines indicate the bubble shapes predicted by Princen. ${ }^{3}$ 


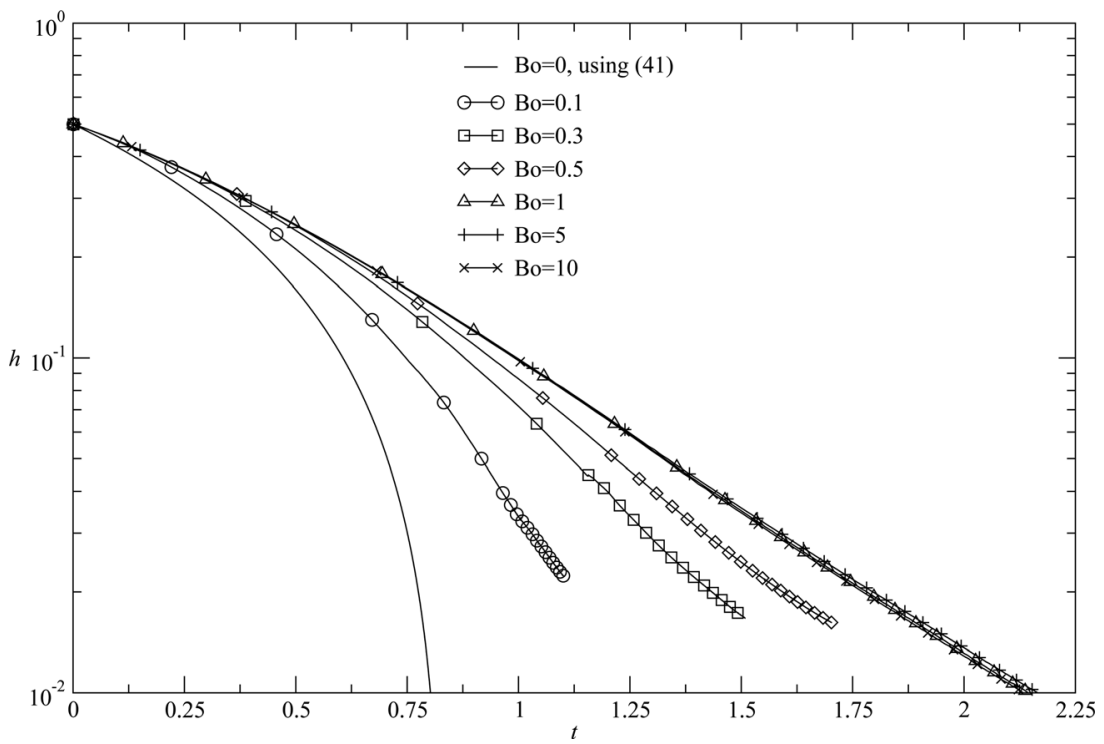

FIG. 10. Film thickness $h$ versus time $t$ at Bo $=0.1$, $0.3,0.5,1,5,10$. The solution for $\mathrm{Bo}=0$ is obtained by the integration of Eq. (41).

\section{Dependence of the film drainage upon the Bond number}

Additional calculations have been carried out for various Bond numbers in the range $[0.1,10]$, using the same initial position and identical discretization. Again, numerical simulations are performed until the dimensionless film thickness reaches a value of order $10^{-2}$ whenever possible. As seen in Fig. 10, where $h$ versus $t$ for various Bond numbers is plotted, it is easy to reach the thickness equal to $10^{-2}$ when $\mathrm{Bo} \gtrsim 1$. In contrast, for smaller Bond number, the $10^{-2}$ accuracy is difficult to reach. Consequently, the numerical computation is stopped before that $h$ decreases below $10^{-2}$.

The use of a log-scale on the $h$ axis suggests that the film drainage behaves as an exponential function of time in the drainage regime, $t \gtrsim \mathcal{O}(1)$. This trend contrasts with the one observed on a viscous drop for which Chi and Leal ${ }^{10}$ proposed a rapid drainage when the drop viscosity is small compared with the liquid viscosity, neutral drainage for equivalent viscosities, and slow drainage for highly viscous drops. Nevertheless, in this last situation, one observes an algebraic evolution of the film drainage as a function of time. For the bubble, the drainage is actually faster because the gas inside and above the fluid interface has no effect on the tangential stress balance. The drainage of a bubble in a highly viscous liquid has been experimentally investigated by Debrégeas et al., ${ }^{8}$ and these authors report an exponential behavior of the film drainage for a bubble suspended in a silicon oil.

As previously suggested, the Bond number has a strong influence on the drainage: the drainage rate increases when Bo decreases (see Fig. 10). When the Bond number is larger or equal to one, the film drainage is very similar. At a first glance, such a trend is amazing. However, since gas does not resist to the flow, the drainage is solely related to the flow in the film. As suggested by the exponential behavior of the film drainage, within the film the flow is a plug flow as it can be shown using lubrication arguments. ${ }^{9}$ In this limit, the drainage is limited by the extensional viscous force, which is more important as the Bond number increases.
As Bo vanishes (for instance for high surface tension or a very small bubble), the fluid interfaces do not deform. In this limit, it is therefore possible to obtain $h$ versus time by employing the exact Stokes flow solution established in Bart ${ }^{38}$ by appealing to the bispherical coordinates procedure. For this purpose, the force balance applied on the bubble can be used. For a spherical bubble with a velocity $\boldsymbol{U}$ normal to the flat interface and a gap $h$, the experienced drag force reads

$$
\boldsymbol{F}_{d}=-6 \pi \mu a \lambda_{d}(h) \boldsymbol{U},
$$

where the drag coefficient $\lambda_{d}$ describes the hydrodynamic interaction between the spherical bubble and the flat fluid interface. This quantity tends to $2 / 3$ when $h$ becomes large. In our axisymmetric case, the force balance between the drag and buoyancy forces, under the dimensionless form, yields

$$
\lambda_{d}(h) \frac{d h}{d t}=-\frac{2}{3} .
$$

The behavior of $\lambda_{d}$ for $h$ small can be obtained using the method proposed by Cox and Brenner. ${ }^{39}$ Taking the drag coefficient given by Bart, ${ }^{38} \lambda_{d}$ behaves as

$$
\lambda_{d}=\frac{2}{3}\left(\gamma_{E}-\frac{\ln h}{2}\right)
$$

with $\gamma_{E} \approx 0.57721$ the Euler's constant. ${ }^{28}$ By virtue of Eq. (42), $\lambda_{d}$ diverges as $h$ tends to zero, but the occurring logarithmic singularity is soft. Moreover, the introduction of the last equation in Eq. (41) gives the following implicit equation for $h$ :

$$
\left(\gamma_{E}+\frac{1}{2}\right) h+h \ln h=\left(\gamma_{E}+\frac{1}{2}\right) h_{0}+h_{0} \ln h_{0}-t,
$$

where $h_{0}$ is the film thickness for $t=0$. This relationship predicts the film rupture in a finite time and mainly explains why the film drainage is faster when the fluid interfaces are undeformed. 
(a)

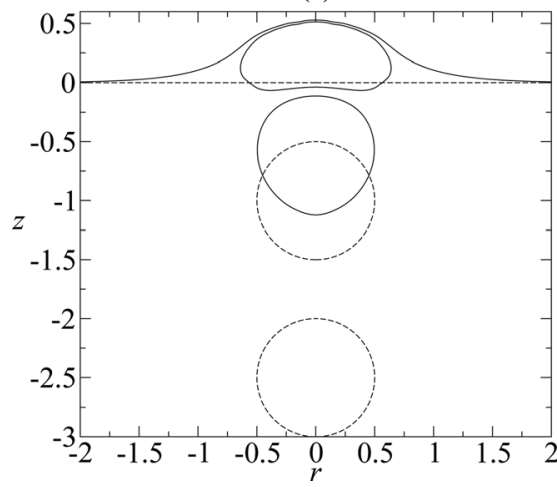

(b)

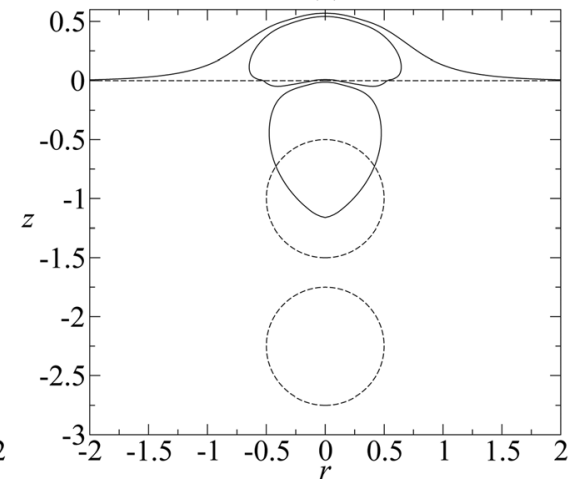

(c)

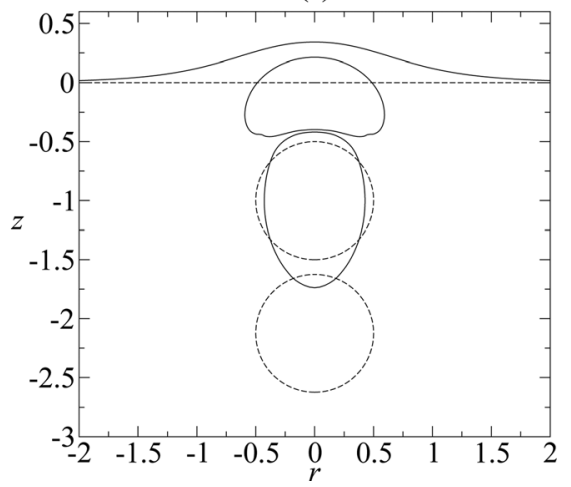

FIG. 11. Bubble shapes for (a) $h_{2}=1 / 2$, (b) $h_{2}=1 / 4$, and (c) $h_{2}=1 / 8$ at $t=0$ and Bo $=1$. Solid lines represent the interfaces at the end of the computation. The dashed lines indicate each initial interface contour.

This conclusion contrasts with this one given by Howell, ${ }^{9}$ who argued that the film behaves as an algebraic function of time when the Bond number is small considering that the gravity force is negligible. However, even if the Bond number is very small, the buoyancy term is still important as we can see in Eq. (41). Consequently, the drainage for a rising bubble takes place under a constant force corresponding to buoyancy effect. This conclusion should be different for a drainage obtained for constant velocity.

\section{Results for two and three bubbles}

So far, our attention has been restricted to the motion of one bubble moving toward a free surface. In this section, the proposed numerical method is used to investigate the axisymmetric drainage of two or three bubbles. Note that adding one or two bubble(s) however increases the number of involved parameters such as each bubble radius, and initial gaps between the bubbles. Whenever possible, each simulation is stopped as soon as the minimum normalized gap between bubbles is order $10^{-2}$.

\section{Sensitivity to the initial bubble-bubble gaps}

The numerical procedure has been applied to the case of two bubbles rising toward a free surface. As depicted in Fig. 1 , the bubble 1 is the closest one to the free surface. The film thickness between the free surface and the first bubble is denoted by $h_{1}$ whilst $h_{2}$ designates the gap between the two bubbles. The sensitivity to the initial gap between the two bubbles is addressed for identical bubbles and $\mathrm{Bo}=1$. For each simulation, the initial value of $h_{1}$ is $1 / 2$ and we use 20 and 25 boundary elements with 4 collocation points $\left(N_{c}=4\right)$ on each bubble and the free surface, respectively.

Fig. 11 shows each bubble shape when the initial distance between the two bubbles is (a) $h_{2}=1 / 2$, (b) $h_{2}=1 / 4$, and (c) $h_{2}=1 / 8$ at $t=0$, respectively. As seen in Fig. 11(a), the first bubble is more deformed than when alone for an equivalent Bond number since the second bubble pushes it toward the free surface. In addition, the film drainage clearly acts between the free surface and the first bubble well before it takes place between the two bubbles.

These trends change when the initial gap $h_{2}$ decreases. For instance, Fig. 11(b) shows that for $h_{2}(t=0)=1 / 4$ the second bubble is this time more deformed and the drainage occurs almost simultaneously above and below the first bubble. As revealed by Fig. 11(c), for $h_{2}(t=0)=1 / 8$, the second bubble is more and more elongated as if it is sucked by the prior bubble, and the drainage occurs first between the two bubbles.

The film drainage is analyzed by plotting in Fig. 12 the film thicknesses $h_{1}$ and $h_{2}$ versus time for each addressed initial condition. As for a single bubble, the film drainage, $h_{1}$, decreases exponentially with time. For initial thickness $h_{2}(t=0)=1 / 2$ and $1 / 4, h_{2}$ stays stable until a time approximately equal to 0.5 and further decreases with the same behavior as $h_{1}$. In Fig. 12(b), when $t \gtrsim 0.75$, the film thicknesses present a similar behavior.

\section{Sensitivity to the bubble sizes}

The influence of bubble sizes is investigated taking two unequal bubbles: a small one with radius $a / 2$ and a big one
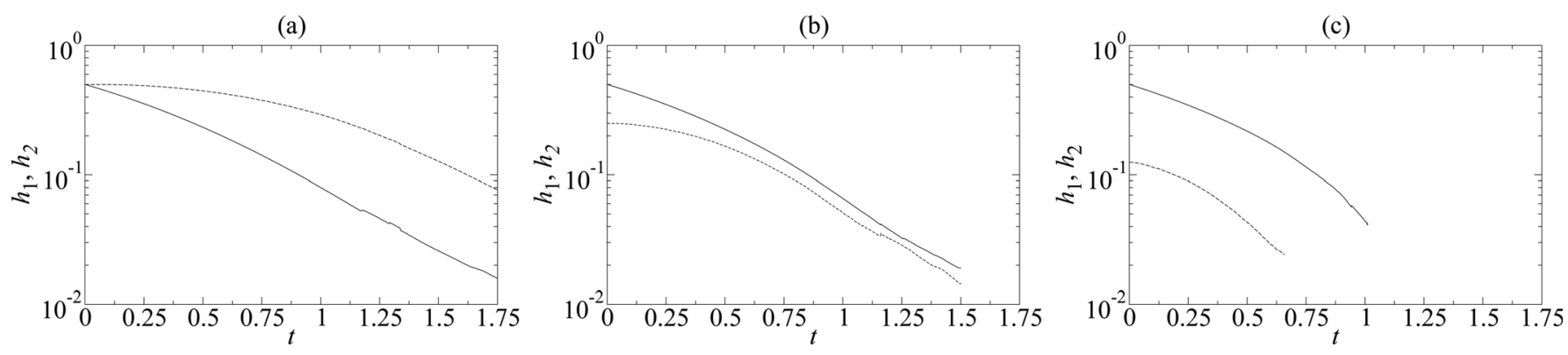

FIG. 12. Film thicknesses $h_{1}$ (solid line) and $h_{2}$ (dashed line) versus time for two bubbles when the initial distance between the two bubbles are (a) $h_{2}=1 / 2$, (b) $h=1 / 4$, and (c) $h=1 / 8$ and $\mathrm{Bo}=1$. 
(a) $\mathrm{Bo}=1$

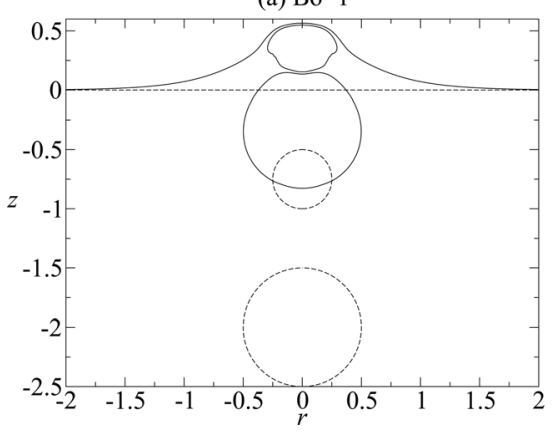

(b) $\mathrm{B}_{0}=4$

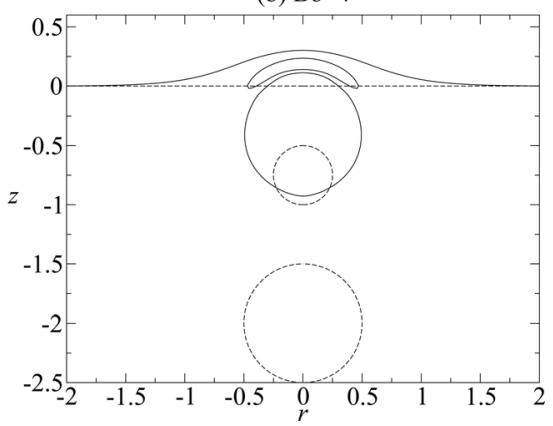

FIG. 13. Bubble shapes for $\mathrm{Bo}=1$ (a) and $\mathrm{Bo}=4$ (b). Solid lines indicate the interfaces at the end of the computation when $\min \left(h_{1}, h_{2}\right) \approx \mathcal{O}\left(10^{-2}\right)$. The dashed lines are initial interfaces. with radius $a$. One sets $\mathrm{Bo}=\rho g a^{2} /(3 \gamma)$ and still takes $2 a$, $U=\rho g a^{2} /(3 \mu)$, and $2 a / U$ as length, velocity, and time scales, respectively. Note that the Bond number based on the small bubble diameter is Bo/4. To study the influence of the Bond number, computations have been done for $\mathrm{Bo}=1$ and 4 . The resulting interface shapes are given in Fig. 13. As seen in Fig. 13(a), bubbles are weakly deformed for $\mathrm{Bo}=1$, especially the (big) bubble 2 . The small bubble 1 is found to exhibit two different curvatures: one on the top driven by the film drainage with the free surface and the second on the bottom due to the approaching second bubble. For $\mathrm{Bo}=4$, different shapes are obtained as seen in Fig. 13(b). For instance, observe that the big bubble is less deformed at $\mathrm{Bo}=4$ than at $\mathrm{Bo}=1$ ! The small bubble for $\mathrm{Bo}=4$ becomes thin and is stretched above and near the big bubble. It therefore screens the interactions between the free surface and the big bubble.

The film thicknesses $h_{1}$ and $h_{2}$ are given in Fig. 14 for these Bond numbers. For Bo $=1$, the film thicknesses present similar behaviors. Observe that $h_{1}$ decreases faster than $h_{2}$ due to the pushing by the second bubble. When $\mathrm{Bo}=4$, the film drainage is smaller than the one observed when $\mathrm{Bo}=1$ both for $h_{1}$ and $h_{2}$. The sensitivity of the film drainage to Bo that we found for a single bubble is thus still valid for two bubbles.

\section{Film drainage sensitivity to the number of bubbles and Bo}

In this subsection, we consider three equal bubbles rising toward the free surface. The distance between successive bubbles is equal to $1 / 2$ as well as the gap between the free surface and the first bubble. Again, computations are actually stopped here at time at which $\min \left(h_{1}, h_{2}, h_{3}\right) \approx 10^{-2}$. First, we examine the influence of the Bond number on the interface shapes by giving in Fig. 15 the free surface and bubble shapes for $\mathrm{Bo}=0.1,0.5$, and 1 .

For the smallest Bond number, all bubbles remain quasispherical, and the free surface is weakly deformed. As the
Bond number increases, the first bubble is more and more deformed and takes a lens form. The second bubble is more and more deformed near its rear (lower side) whereas the last bubble undergoes the sucking of the previous bubble. This behavior is similar to the one observed for two bubbles. Actually, the last bubble is only sucked by the preceding bubble leading to the elongation.

The influence of the bubble number on the film drainage between the free surface and the first bubble is addressed by plotting in Fig. 16 for one, two, and three bubbles the film thickness $h_{1}$ versus time $t$ still for $\mathrm{Bo}=0.1,0.5$, and 1 .

As observed for one bubble, and two and three bubbles, the film thickness $h_{1}$ exhibits an exponential decay beyond the free rising regime of the first bubble. Adding one bubble increases the film drainage as clearly shown in Fig. 16(a) when $\mathrm{Bo}=0.1$. Actually, at low Bond number, a second bubble has a very small influence on the shape of the first bubble located near the free surface but has a strong influence on the film drainage taking place between this first bubble and the free surface interface. This is because the second bubble pushes the first one toward the free surface, thereby initiating earlier the film drainage than in the case of a single bubble. In addition, the increase of the thinning rate with two bubbles is less pronounced when the Bond number increases, and the presence of a third bubble only weakly affects the thinning rate when the Bond number exceeds 0.5 .

\section{CONCLUSIONS}

This work examines the axisymmetric motion of a bubble chain rising toward a free surface. For this purpose, a relevant boundary-integral approach has been both proposed and carefully implemented. The numerical procedure is based on a discretization of boundaries, using discontinuous elements with variables interpolated using Lagrangian polynomials. Furthermore, a well-posed regular linear system is obtained by using a discrete Wielandt's deflation.
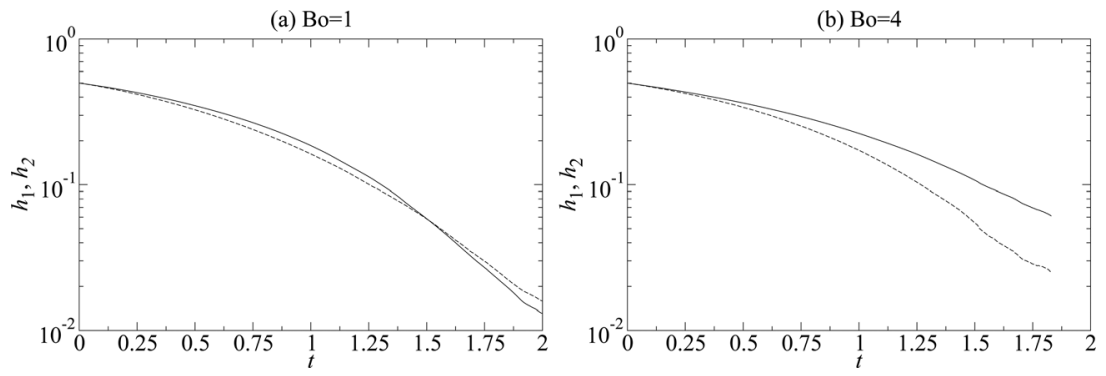

FIG. 14. Film thicknesses $h_{1}$ (solid line) and $h_{2}$ (dashed line) versus time for the two bubbles for $\mathrm{Bo}=1$ (a) and $\mathrm{Bo}=4(\mathrm{~b})$. 
(a) $\mathrm{Bo}=0.1$

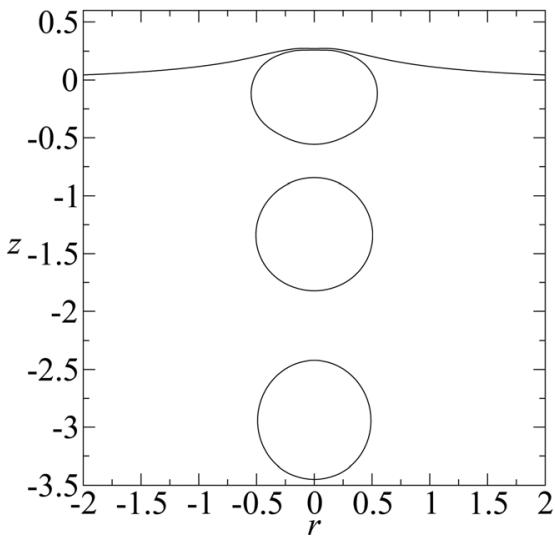

(b) $\mathrm{Bo}=0.5$

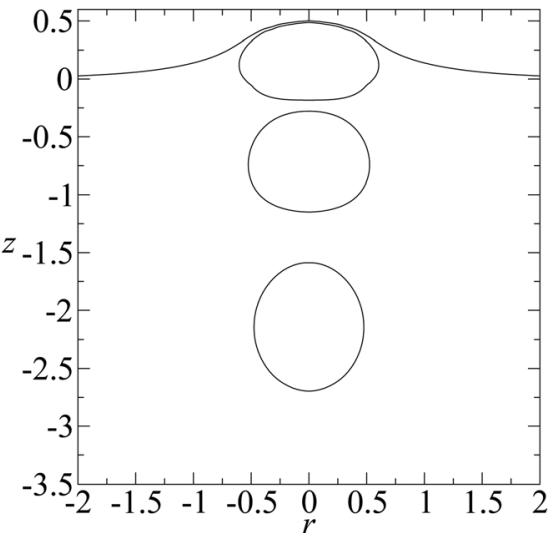

(c) $\mathrm{Bo}=1$

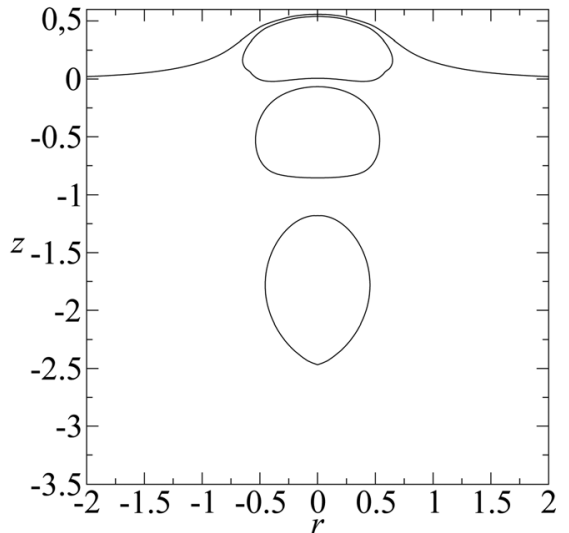

FIG. 15. Interface shapes for three rising bubbles at (a) $\mathrm{Bo}=0.1$ and (b) $\mathrm{Bo}=0.5$.

The numerical procedure has been tested against some integral identities verified by the Green functions, the rise of a bubble in an infinite media, and the computation of discrete eigenvalues of the double-layer potential of the Stokes equations.

The rise of a bubble toward a free surface has been investigated, with a special attention paid to the film drainage between the bubble interface and the free surface. When close to the free interface, the bubble is found to adapt a quasi-static shape. The obtained bubble shapes have been compared with the results given by Princen. ${ }^{3}$ The thinning rate appears to behave as an exponential function of time, in agreement with prior experimental results obtained by Debrégeas et al. ${ }^{8}$ The basic influence of the Bond number (ratio of the gravity force to the surface tension force) has been clearly revealed by the computation. More precisely, at small Bond number, the drainage is fast, mainly because of the weak deformation of fluid interfaces. For a sufficiently large Bond number (larger than 1 with a definition used in this article), the film drainage becomes independent of the Bond number.

Computations with one or two additional bubble(s) have also been performed. The initial distance between bubbles is found to affect mainly the deformation of the last bubble, because of the sucking of the first bubble. However, introducing one or two bubble(s) does not dramatically change the general behavior of the film drainage of the first bubble, which still exhibits an exponential thinning. Finally, the film drainage is seen to decrease with the Bond number.
As explained in this work, when the Bond number Bo is zero, the collapse of the liquid film between the bubble interface and the free surface occurs in a finite time obtained by solving Eq. (41). For a weak and non-zero Bond number, it is this time necessary to approximate the drag force exerted on the bubble for a slightly deformed bubble and fluid interface, in order to gain a modified equation (41) and the thin film collapse time. Such a challenging task is postponed to future investigations.

\section{APPENDIX A: SIMPLE AND DOUBLE-LAYER OPERATORS IN AXISYMMETRIC FORMULATION}

For $\boldsymbol{x}_{0}$ and $\boldsymbol{x}$ having cylindrical coordinates $\left(r_{0}, z_{0}\right)$ and $(r, z)$, respectively, one gets ${ }^{20}$

$$
\begin{aligned}
B_{z z}\left(\boldsymbol{x}, \boldsymbol{x}_{0}\right) & =r\left(I_{10}+\hat{z}^{2} I_{30}\right), \quad B_{z r}\left(\boldsymbol{x}, \boldsymbol{x}_{0}\right)=r \hat{z}\left(r I_{30}-r_{0} I_{31}\right), \\
B_{r z}\left(\boldsymbol{x}, \boldsymbol{x}_{0}\right) & =r \hat{z}\left(r I_{31}-r_{0} I_{30}\right), \\
B_{r r}\left(\boldsymbol{x}, \boldsymbol{x}_{0}\right) & =r\left[I_{11}+\left(r^{2}+r_{0}^{2}\right) I_{31}-r_{0} r\left(I_{30}+I_{32}\right)\right]
\end{aligned}
$$

with $\hat{z}=z-z_{0}$ for the single-layer matrix and, setting $\boldsymbol{n}(\boldsymbol{x})=n_{r} \boldsymbol{e}_{r}+n_{z} \boldsymbol{e}_{z}$, the relations

$$
\begin{aligned}
C_{z z}\left(\boldsymbol{x}, \boldsymbol{x}_{0}\right)= & -6 r \hat{z}^{2}\left[\hat{z} I_{50} n_{z}+\left(r I_{50}-r_{0} I_{51}\right) n_{r}\right], \\
C_{z r}\left(\boldsymbol{x}, \boldsymbol{x}_{0}\right)= & -6 r \hat{z}\left[\hat{z}\left(r I_{50}-r_{0} I_{51}\right) n_{z}\right. \\
& \left.+\left(r_{0}^{2} I_{52}+r^{2} I_{50}-2 r r_{0} I_{51}\right) n_{r}\right]
\end{aligned}
$$
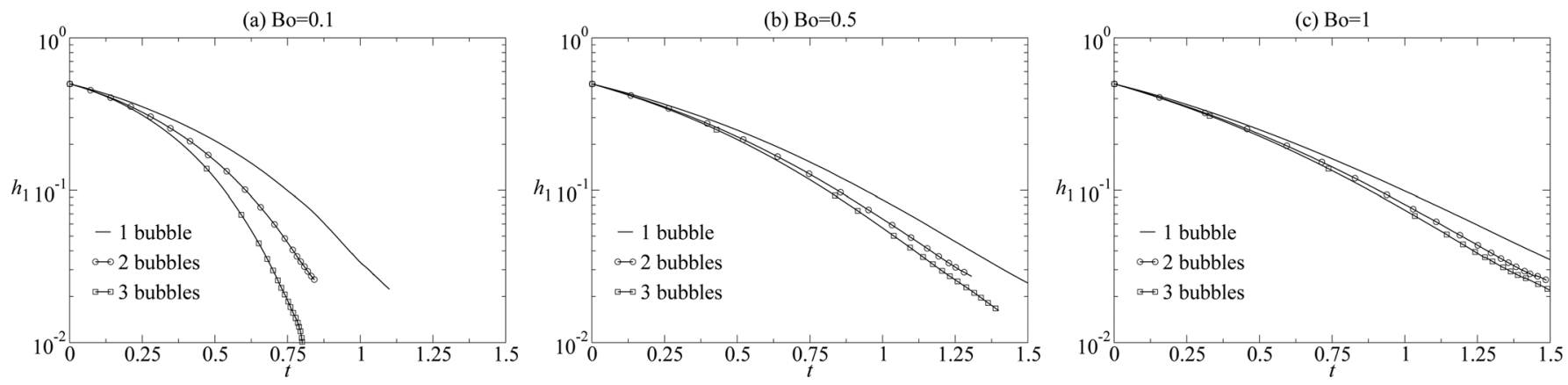

FIG. 16. Film thickness $h_{1}$ versus time for one, two, and three bubbles for $\mathrm{Bo}=0.1$ (a), 0.5 (b), and 1 (c). 


$$
\begin{aligned}
C_{r z}\left(\boldsymbol{x}, \boldsymbol{x}_{0}\right)= & -6 r \hat{z}\left\{\hat{z}\left(r I_{51}-r_{0} I_{50}\right) n_{z}\right. \\
& \left.+\left[\left(r^{2}+r_{0}^{2}\right) I_{51}-r r_{0}\left(I_{50}+I_{52}\right)\right] n_{r}\right\}, \\
C_{r r}\left(\boldsymbol{x}, \boldsymbol{x}_{0}\right)= & -6 r\left\{\hat{z}\left[\left(r^{2}+r_{0}^{2}\right) I_{51}-r r_{0}\left(I_{50}+I_{52}\right)\right] n_{z}\right. \\
& +\left[r^{3} I_{51}-r^{2} r_{0}\left(I_{50}+2 I_{52}\right)\right. \\
& \left.\left.+r_{0}^{2}\left(I_{53}+2 I_{51}\right)-r_{0}^{3} I_{52}\right] n_{r}\right\},
\end{aligned}
$$

for the double-layer matrix with previous quantities $I_{m n}$ defined as

$$
\begin{aligned}
I_{m n}\left(r, r_{0}, \hat{z}\right) & =\frac{4 k^{m}}{\left(4 r r_{0}\right)^{m / 2}} \int_{0}^{\pi / 2} \frac{\left(2 \cos ^{2} \phi-1\right)^{n}}{\left(1-k^{2} \cos ^{2} \phi\right)^{m / 2}} d \phi, \\
k^{2} & =\frac{4 r r_{0}}{\hat{z}^{2}+\left(r+r_{0}\right)^{2}} .
\end{aligned}
$$

\section{APPENDIX B: MATERIAL FOR THE NUMERICAL IMPLEMENTATION}

At a point $\boldsymbol{x}^{j_{e}}$ with coordinate $\zeta$ in the segment $[-1,1]$ and located on the boundary element $j_{e}$, we denote by $l_{j_{e}}^{\prime}(\zeta)=\left\{z_{j_{e}}^{\prime 2}(\zeta)+r_{j_{e}}^{\prime 2}(\zeta)\right\}^{1 / 2}$ the differential arc length where primes indicate differentiation. One then gets

$$
\begin{aligned}
& n_{z}\left(\boldsymbol{x}^{j_{e}}\right)=-\frac{r_{j_{e}}^{\prime}(\zeta)}{l_{j_{e}}^{\prime}(\zeta)}, \quad n_{r}\left(\boldsymbol{x}^{j_{e}}\right)=\frac{z_{j_{e}}^{\prime}(\zeta)}{l_{j_{e}}^{\prime}(\zeta)}, \\
& \nabla_{S} \cdot \boldsymbol{n}\left(\boldsymbol{x}^{j_{e}}\right)=\frac{z_{j_{e}}^{\prime}}{r_{i_{e}} \sqrt{z_{j_{e}}^{\prime \prime}+r_{j_{e}}^{\prime \prime}}}+\frac{r_{j_{e}}^{\prime} z_{j_{e}}^{\prime \prime}-z_{j_{e}}^{\prime} r_{j_{e}}^{\prime \prime}}{\left(z_{j_{e}}^{\prime \prime}+r_{j_{e}}^{\prime \prime}\right)^{3 / 2}}
\end{aligned}
$$

Furthermore, the matrices $\boldsymbol{B}$ and $\boldsymbol{C}$ occurring in Eq. (23) consist of $N_{e} \times N_{e}$ square block of order $2 N_{c} \times 2 N_{c}$ with coefficients

$$
\begin{gathered}
B_{\alpha \beta ; i_{c} j_{c}}^{i_{e} j_{e_{e}}}=-\frac{1}{4 \pi} \int_{-1}^{1} B_{\alpha \beta}\left(\boldsymbol{x}^{j_{e}}, \boldsymbol{x}_{i_{c}}^{i_{e}}\right) L_{j_{c}}(\zeta) l_{j_{e}}^{\prime}(\zeta) d \zeta \\
C_{\alpha \beta ; i_{c} j_{c}}^{i_{e} j_{e}}=\frac{1}{4 \pi} \int_{-1}^{1} C_{\alpha \beta}\left(\boldsymbol{x}^{j_{e}}, \boldsymbol{x}_{i_{c}}^{i_{e_{c}}}\right) L_{j_{c}}(\zeta) l_{j_{e}}^{\prime}(\zeta) d \zeta \text { for } i_{e} \neq j_{e}, \\
C_{\alpha \beta ; i_{c} j_{c}}^{i_{i_{i} i_{e}}}=\frac{1}{4 \pi} \int_{-1}^{1} C_{\alpha \beta}\left(\boldsymbol{x}^{i_{e}}, \boldsymbol{x}_{i_{c}}^{i_{e}}\right) L_{j_{c}}(\zeta) l_{i_{e}}^{\prime}(\zeta) d \zeta
\end{gathered}
$$

where $\boldsymbol{x}_{i_{c}}^{i_{e}}$ is the collocation point (with label $i_{c}$ on the boundary element $i_{e}$ ) at which the discretized boundary-integral equation (21) is enforced. Two cases then occur when computing the integrals (B2)-(B4):

(i) Regular integrals: This is not only the case when $\boldsymbol{x}_{i_{c}}^{i_{e}}$ is not located on the boundary element $j_{e}$. Indeed, if $\boldsymbol{x}_{i_{c}}^{i_{e}}$ with intrinsic coordinate $\zeta_{i c}$ in the segment $[-1,1]$, belongs to the boundary element $j_{e}$ the off-diagonal components, $B_{z r}\left(\boldsymbol{x}^{i_{e}}, \boldsymbol{x}_{i_{c}}^{i_{e}}\right)$ and $B_{r z}\left(\boldsymbol{x}^{i_{e}}, \boldsymbol{x}_{i_{c}}^{i_{e}}\right)$ are regular $^{20}$ and, as $\zeta \rightarrow \zeta_{i c}$, one gets

$$
\begin{aligned}
C_{z z}\left(\boldsymbol{x}^{i_{e}}, \boldsymbol{x}_{i_{c}}^{i_{e}}\right)= & -\frac{8 z_{i_{e}}^{\prime 2}\left(\zeta_{i_{c}}\right)}{l_{i_{e}}^{\prime \prime}\left(\zeta_{i_{c}}\right)} \frac{z_{i_{e}}^{\prime}\left(\zeta_{i_{c}}\right) n_{z}+r_{i_{e}}^{\prime}\left(\zeta_{i_{c}}\right) n_{r}}{\zeta-\zeta_{i_{c}}}, \\
C_{z r}\left(\boldsymbol{x}^{i_{e}}, \boldsymbol{x}_{i_{c}}^{i_{e}}\right)= & C_{r z}=-\left[\frac{8 z_{i_{e}}^{\prime}\left(\zeta_{i_{c}}\right) r_{i_{e}}^{\prime}\left(\zeta_{i_{c}}\right)}{l_{i_{e}}^{\prime 4}\left(\zeta_{i_{c}}\right)}\right] \\
& \times\left[\frac{z_{i_{e}}^{\prime}\left(\zeta_{i_{c}}\right) n_{z}+r_{i_{e}}^{\prime}\left(\zeta_{i_{c}}\right) n_{r}}{\zeta-\zeta_{i_{c}}}\right],
\end{aligned}
$$

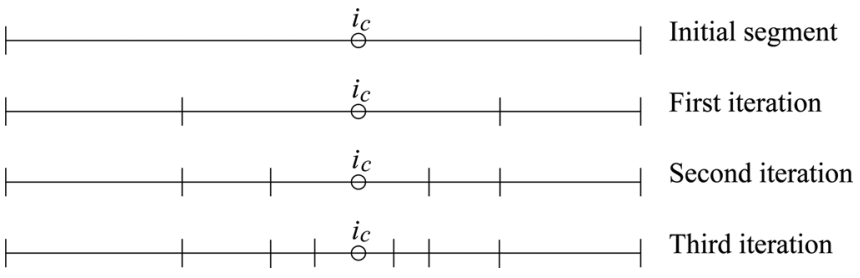

FIG. 17. Non-uniform refinement of the integration grid on the segment $[-1,1]$ near the collocation point $i_{c}$ when the number of partitions is equal to 2 .

$$
C_{r r}\left(\boldsymbol{x}^{i_{e}}, \boldsymbol{x}_{i_{c}}^{i_{e}}\right)=-\frac{8 r_{i_{e}}^{\prime 2}\left(\zeta_{i_{c}}\right)}{l_{i_{e}}^{\prime \prime}\left(\zeta_{i_{c}}\right)} \frac{z_{i_{e}}^{\prime}\left(\zeta_{i_{c}}\right) n_{z}+r_{i_{e}}^{\prime}\left(\zeta_{i_{c}}\right) n_{r}}{\zeta-\zeta_{i_{c}}} .
$$

Exploiting (B1) then immediately shows that, as $\zeta$ $\rightarrow \zeta_{i c}$

$$
\begin{aligned}
& z_{i_{e}}^{\prime}\left(\zeta_{i_{c}}\right) n_{z}+r_{i_{e}}^{\prime}\left(\zeta_{i_{c}}\right) n_{r} \\
& \quad=\frac{r_{i_{e}}^{\prime}\left(\zeta_{i_{c}}\right) z_{i_{e}}^{\prime \prime}\left(\zeta_{i_{c}}\right)-r_{i_{e}}^{\prime \prime}\left(\zeta_{i_{c}}\right) z_{i_{e}}^{\prime}\left(\zeta_{i_{c}}\right)}{l_{i_{e}}^{\prime}\left(\zeta_{i_{c}}\right)}\left(\zeta-\zeta_{i_{c}}\right) .
\end{aligned}
$$

Accordingly, the integrals (B3)-(B4) are also regular ones.

(ii) Weakly singular integrals: This happens only for integrals (B2) when $\alpha=\beta$ and $\boldsymbol{x}_{i_{c}}^{i_{e}}$ is located on the boundary element $j_{e}$. This time

$$
\begin{aligned}
& B_{r r}\left(\boldsymbol{x}^{i_{e}}, \boldsymbol{x}_{i_{c}}^{i_{e}}\right) \sim B_{z z}\left(\boldsymbol{x}^{i_{e}}, \boldsymbol{x}_{i_{c}}^{i_{e}}\right) \sim 2 \ln \left[\frac{8 r_{i_{e}}\left(\zeta_{i_{c}}\right)}{l_{i_{e}}^{\prime}\left(\zeta_{i_{c}}\right)\left|\zeta-\zeta_{i_{c}}\right|}\right] \\
& \quad \text { as } \zeta \rightarrow \zeta_{i_{c}},
\end{aligned}
$$

and we adopt the isolation and analytical integration of the above weakly singular logarithmic term as explained in Pozrikidis, ${ }^{20}$ therefore, finally ending with the numerical evaluation of two regular integrals over the segments $\left[-1, \xi_{i c}\right]$ and $\left[\xi_{i c}, 1\right]$.

Each regular integral encountered in previous cases (i)-(ii) is iteratively computed by using the Voutsinas and Bergeles ${ }^{29}$ procedure which here consists in dividing in case (i) the segment $[-1,1]$ into equal or unequal subsegments when the point $\boldsymbol{x}_{i_{c}}^{i_{e}}$ is not too close or close the boundary element $j_{e}$ and also using, as illustrated in Fig. 17, a non-uniform refinement of the segments $\left[-1, \xi_{i c}\right]$ and $\left[\xi_{i c}, 1\right]$ in case (ii).

${ }^{1}$ M. Manga, "The motion of deformable drops and bubbles at low Reynolds numbers: Application to selected problems in geology and geophysics," Ph.D. thesis, Harvard University, 1994.

${ }^{2}$ J. Kappel, R. Conradt, and H. Scholze, "Foaming behaviour on glass melts," Glastech. Ber. 60, 189 (1987).

${ }^{3}$ H. M. Princen, "Shape of a fluid drop at a liquid-liquid interface," J. Colloid Interface Sci. 18, 178 (1963).

${ }^{4} \mathrm{~S}$. Hartland, "The coalescence of a liquid drop at a liquid-liquid interface. Part I: Drop shape,” Trans. Inst. Chem. Eng. 45, T97 (1967).

${ }^{5} \mathrm{~S}$. Hartland, "The coalescence of a liquid drop at a liquid-liquid interface. Part II: Film thickness,” Trans. Inst. Chem. Eng. 45, T102 (1967).

${ }^{6} \mathrm{~S}$. Hartland, "The profile of the draining film between a fluid drop and a deformable fluid-liquid interface," Chem. Eng. J. 1, 67 (1970).

${ }^{7}$ A. F. Jones and S. D. R. Wilson, "The film drainage problem in droplet coalescence," J. Fluid Mech. 87, 263 (1978). 
${ }^{8}$ G. Debrégeas, P.-G. de Gennes, and F. Brochard-Wyart, "The life and death of 'bare' viscous bubbles," Science 279, 1704 (1998).

${ }^{9}$ P. D. Howell, "The draining of a two-dimensional bubble," J. Eng. Math. 35, 251 (1999).

${ }^{10}$ B. K. Chi and L. G. Leal, "A theoretical study of the motion of a viscous drop toward a fluid interface at low Reynolds number," J. Fluid Mech. 201, 123 (1989).

${ }^{11}$ C. W. Hirt and B. D. Nichols, "Volume of fluid (VOF) method for the dynamics of free boundaries," J. Comput. Phys. 39, 201 (1981).

${ }^{12}$ M. Sussman, P. Smereka, and S. Osher, "A level set approach for computing solutions to incompressible two-phase flow," J. Computat. Phys. 114, 146 (1994).

${ }^{13}$ A. O. Unverdi and G. Tryggvason, "A front-tracking method for viscous, incompressible, multi-fluid flows," J. Computat. Phys. 100, 25 (1992).

${ }^{14} \mathrm{M}$. Bonnet, Boundary Integral Method for Solid and Fluid (Springer, Berlin, 1995).

${ }^{15}$ H. A. Lorentz, "Ein allgemeiner Satz, die Bewegung einer reibenden Flüssigkeit betreffend, nebst einigen Anwendungen desselben," in Abhandlungen über Theoretische Physik, B. G. Teubner, Berlin, 1907, pp. 23-42.

${ }^{16}$ F. K. G. Odqvist, "Über die Randwertaufgaben der Hydrodynamik zäher Flüssigkeiten,” Math. Z. 32, 329 (1930).

${ }^{17}$ O. A. Ladyzhenskaya, The Mathematical Theory of Viscous Incompressible Flow (Gordon and Breach, New York, 1963).

${ }^{18}$ G. K. Youngren and A. Acrivos, "Stokes flow past a particle of arbitrary shape: A numerical method of solution," J. Fluid Mech. 69, 377 (1975).

${ }^{19}$ J. M. Rallison and A. Acrivos, "A numerical study of the deformation and burst of a viscous drop in extensional flow," J. Fluid Mech. 89, 191 (1978).

${ }^{20} \mathrm{C}$. Pozrikidis, Boundary Integral and Singularity Methods for Linearized Viscous Flow (Cambridge University Press, Cambridge, England, 1992).

${ }^{21}$ S. Kim and S. J. Karrila, Microhydrodynamics. Principles and Selected Applications (Dover, New York, 2005).

${ }^{22} \mathrm{R}$. Aris, Vectors, Tensors and the Basic Equation of Fluid Mechanics (Dover, New York, 1962).

${ }^{23}$ J. Hadamard, Le problème de Cauchy et les équations aux dérivées partielles linéaires hyperboliques (Hermann \& Cie, Paris, 1923).

${ }^{24}$ V. D. Kupradze, Dynamical Problems in Elasticity. In Progress in Solid Mechanics (North-Holland, New York, 1963).
${ }^{25} \mathrm{M}$. Loewenberg and E. J. Hinch, "Numerical simulation of a concentrated emulsion in shear flow,” J. Fluid Mech. 321, 395 (1996).

${ }^{26}$ A. Z. Zinchenko, M. A. Rother, and R. H. Davis, "A novel boundaryintegral algorithm for viscous interaction of deformable drops," Phys. Fluids 9,1493 (1997).

${ }^{27}$ G. P. Muldowney and J. J. L. Higdon, "A spectral boundary element approach to three-dimensional Stokes flow,” J. Fluid Mech. 298, 167 (1995).

${ }^{28} \mathrm{M}$. Abramowitz and I. A. Stegun, Handbook of Mathematical Functions (Dover, New York, 1965).

${ }^{29} \mathrm{~S}$. Voutsinas and G. Bergeles, "Numerical calculation of singular integrals appearing in three-dimensional potential flow problems," Appl. Math. Model. 14, 618 (1990).

${ }^{30}$ J. Stoer and R. Bulirsch, Introduction to Numerical Analysis (SpringerVerlag, New York, 1993).

${ }^{31}$ W. H. Press, B. P. Flannery, S. A. Teukolsky, and W. T. Vetterling, Numerical Recipes in C. The Art of Scientific Computing (Cambridge University Press, Cambridge, England, 1988).

${ }^{32}$ For our numerical computations, $\varepsilon=\mathcal{O}\left(10^{-5}\right)$.

${ }^{33}$ T. Taylor and A. Acrivos, "On the deformation and drag of a falling viscous drop at low Reynolds number,” J. Fluid Mech. 18, 466 (1964).

${ }^{34}$ F. Y. Pan and A. Acrivos, "Shape of a drop or bubble at low Reynolds number," Ind. Eng. Chem. Fundam. 7, 227 (1968).

${ }^{35}$ J. Hadamard, "Mouvement permanent lent d'une sphre liquide et visqueuse dans un liquide visqueux," C. R. Acad. Sci. Paris 152, 1735 (1911).

${ }^{36}$ W. Rybczynski, "Über die fortschreitende Bewegung einer flüssigen Kugel in einem zähen Medium," Bull. de l'Acad. des Sci. de Cracovie, Ser. A 1, 40 (1911).

${ }^{37}$ For $\mathrm{Bo}=\mathcal{O}(1)$, the surface tension is large enough so that the surfaces are weakly deformed. In contrast, for $\mathrm{Bo}=\mathcal{O}(10)$, the bubble and the free surface experience deformations that are more difficult to accurately compute. We thus propose computations for this regime.

${ }^{38}$ E. Bart, "The slow unsteady settling of a fluid sphere toward a flat fluid interface," Chem. Eng. Sci. 23, 193 (1968).

${ }^{39}$ R. G. Cox and H. Brenner, "The slow motion of a sphere through a viscous fluid towards a plane surface. II. Small gap widths, including inertial effects," Chem. Eng. Sci. 22, 1753 (1967). 